\title{
Black frole solutions in massive gravity
}

\author{
Michael V. Bebronne \\ Service de Physique Théorique, Université Libre de Bruxelles (ULB), \\ CP225, Boulevard du Triomphe, 1050 Brussels, Belgium.
}

Peter G. Tinyakov

Service de Physique Théorique, Université Libre de Bruxelles (ULB), CP225, Boulevard du Triomphe, 1050 Brussels, Belgium.

Institute for Nuclear Research of the Russian Academy of Sciences, 60th October Anniversary Prospect, 7a, 117312 Moscow, Russia.

\begin{abstract}
A B S T R A C T
The static vacuum spherically symmetric solutions in massive gravity are obtained both analytically and numerically. The solutions depend on two parameters (integration constants): the mass $M$ (or, equivalently, the Schwarzschild radius), and an additional parameter, the "scalar charge" $S$. At zero value of $S$ and positive mass the standard Schwarzschild black hole solutions are recovered. Depending on the parameters of the model and the signs of $M$ and $S$, the solutions may or may not have horizon. Those with the horizon describe modified black holes provided they are stable against small perturbations. In the analytically solvable example, the modified black hole solutions may have both attractive and repulsive (anti-gravitating) behavior at large distances. At intermediate distances the gravitational potential of a modified black hole may mimics the presence of dark matter. Modified black hole solutions are also found numerically in more realistic massive gravity models which are attractors of the cosmological evolution.
\end{abstract}

ULB reference:

ULB-TH/09-05

Key words:

Massive gravity, Spherically symmetric solutions, Black holes

\section{Introduction}

Recent advances in observational cosmology [1, 2, 3] have revived interest in alternative theories of gravity in which the gravitational interaction is modified in the infrared domain and which could - potentially — explain the accelerated expansion of the Universe without introducing the dark energy and matter components. Theoretical consistency and existing experimental data impose severe constraints on such models. Different approaches to the problem have been discussed in the literature (see, e.g., Refs. [4, 5, 6, 7, 8, 9, 10, 11], and Refs. [12, 13, 14] for reviews). One of them employs spontaneous breaking of Lorentz symmetry by space-time dependent condensates of scalar fields [15, 16] coupled to gravity in a covariant way via a derivative coupling. The resulting theory may have a non-pathological perturbative behavior about the broken vacuum $[17,16]$ and exhibit modifications of gravitational interactions at large scales (see Sect. 2 for more details). In particular, graviton may acquire a non-zero mass, which is the reason to call these models massive gravity models.

Existing experimental data constrain the mass of the graviton and other parameters of massive gravity models. Absence of Lorentz invariance makes the constraints weaker than one would expect in a Lorentz-invariant theory: the Newton's potential remains unmodified in the linear approximation despite non-zero graviton mass [18], so that Solar system constraints are satisfied for rather large masses. The constraint on the mass of the graviton comes from the emission of gravitational waves by binary pulsars which is responsible for their spin-down [19]. Consistency of observations with GR requires the graviton mass to be smaller than the inverse period of orbital motion of the binary system, that is [20]

$$
m \lesssim 10^{-19} \mathrm{eV}
$$

Standard cosmological solutions may be reproduced in massive gravity [18]. Further constraints on the parameters of the model are imposed by the growth of perturbations and structure formation in the post-inflationary epoch [21].

In General Relativity (GR), a crucial role is played by the spherically symmetric vacuum solution to the Einstein equations - the Schwarzschild solution. This role is twofold: First, this solution describes the metric outside of spherical non-rotating bodies and gives rise, in the weak field limit, to the Newtonian gravity. It provides therefore a useful approximation in many astrophysical situations.

Second, the Schwarzschild solution describes the result of a gravitational collapse, the black hole. Although the existence of black holes has not yet been directly confirmed, there exists an indirect evidence that some of binary stellar systems contain black holes as one of the companion [22, 23], and that many galaxies, including the Milky Way, harbor super-massive black holes in their centers [24, 25]. It is conceivable that black holes will be directly observed in the near future, and that their properties, including the 
metric configuration near the horizon, will be quantitatively tested [26], thus providing a probe of GR in a fully non-linear regime.

The Schwarzschild metric, together with properly arranged scalar fields is a solution to Einstein equations in massive gravity as well [27]. However, the properties of black holes are, in general, expected to be different. In particular, rotating black holes are certainly modified, and, more generally, black holes are expected to have hair [27]. The possible existence of black hole hair in massive gravity models suggests that there might exist spherically symmetric solutions other than the Schwarzschild one. In this paper we construct explicit examples of such solutions.

We found a new class of vacuum spherically-symmetric solutions in massive gravity which depend, in addition to the mass $M$ (equivalently, the Schwarzschild radius), on one more parameter which we call the "scalar charge" $S$. At zero value of this parameter the standard Schwarzschild solution is recovered, while at non-zero values of $S$ the Schwarzschild metric gets modified. The modified solution is non-linear at all distances; it cannot be obtained in the linear approximation. Similar phenomenon has been previously found in the context of bi-gravity models [28]. The new solutions may have event horizons and are, therefore, candidates for modified black holes. We found both analytical and numerical examples of such modified black holes.

The analytical solutions found in a particular massive gravity model show a variety of different behaviors. Depending on the parameters of the model, the deviation of the metric from the Minkowski one may decay at infinity as $1 / r$ or slower. The solutions thus may have finite or infinite ADM [29] mass, respectively. In the case of a finite mass, this mass may be positive or negative depending on the sign of $M$. In either case the singularity at the origin may be hidden by the horizon. The solutions with negative mass exhibit an anti-gravitating behavior at large distances from the center.

The modified gravitational potential may decay slower than $1 / r$ in a certain distance range. Such a behavior would mimic the effect of the dark matter. Interestingly, the modification depends not only on the parameters of the model, but also on the scalar charge and the mass of the solution. Thus, the apparent amount of the "dark matter" may be different for objects of the same mass, in contrast with other models of modified gravity.

This paper is organized as follows. In Sect. 2 we briefly review the massive gravity model and summarize previous results about the Newtonian potential in this model. In Sect. 3 we present the spherically symmetric ansatz and reduce the Einstein equations to four ordinary differential equations. In Sect. 4 we choose a particular model and find analytical solutions to the Einstein equations in this model. In Sect. 5 we demonstrate, by numerical computation, that modified black hole solutions exist in more realistic massive gravity models which are attractors of the cosmological evolution. Finally, Sect. 6 contains the summary and discussion of our results.

\section{The massive gravity model}

In this paper we consider the massive gravity model described by the following action [16],

$$
\mathcal{S}=\int \mathrm{d} x^{4} \sqrt{-g}\left[-\mathrm{M}_{\mathrm{pl}}^{2} \mathcal{R}+\mathcal{L}_{\mathrm{m}}+\Lambda^{4} \mathcal{F}\right] .
$$

The first two terms are the curvature and the Lagrangian of the minimally coupled ordinary matter; they comprise the standard GR action. The third term describes four scalar fields $\phi^{0}, \phi^{i}$ whose space-time dependent vacuum expectation values break spontaneously the Lorentz symmetry. These fields are minimally coupled to gravity through a derivative coupling; they will be referred to as the Goldstone fields. We consider the functions $\mathcal{F}$ which depend on two particular combinations of the derivatives of the Goldstone fields, $\mathcal{F}=\mathcal{F}\left(X, W^{i j}\right)$, where

$$
\begin{aligned}
X & =\frac{\partial^{\mu} \phi^{0} \partial_{\mu} \phi^{0}}{\Lambda^{4}}, \\
W^{i j} & =\frac{\partial^{\mu} \phi^{i} \partial_{\mu} \phi^{j}}{\Lambda^{4}}-\frac{\partial^{\mu} \phi^{i} \partial_{\mu} \phi^{0} \partial^{\nu} \phi^{j} \partial_{\nu} \phi^{0}}{\Lambda^{8} X} .
\end{aligned}
$$

The constant $\Lambda$ has the dimension of mass. The model is understood as the low-energy effective theory valid below the scale $\Lambda$.

The vacuum configuration has the form

$$
g_{\mu \nu}=\eta_{\mu \nu}, \quad \phi^{0}=a \Lambda^{2} t, \quad \phi^{i}=b \Lambda^{2} x^{i},
$$

where $a$ and $b$ are two constants determined by the requirement that the energy-momentum tensor associated with the four scalar fields vanishes in the Minkowski background. The configuration (2) is, therefore, a solution to the Einstein equations. The constants $a$ and $b$ may be set to one by the redefinition of fields, which we assume to be the case in what follows.

For functions $\mathcal{F}$ which are invariant under rotations of the Goldstone fields $\phi^{i}$ in the internal space (i.e., those depending on $W^{i j}$ through three combinations $w_{n}=\operatorname{Tr} W^{n}$, $n=1,2,3$ ), the background (2) preserves the rotational symmetry. The Lorentz symmetry is, in general, broken.

The action of the Goldstone fields with functions $\mathcal{F}$ depending only on $X$ and $W^{i j}$ is invariant under the following symmetry,

$$
\phi^{i} \rightarrow \phi^{i}+\chi^{i}\left(\phi^{0}\right),
$$

where $\chi^{i}$ are arbitrary functions of $\phi^{0}$. Because of this symmetry, the behavior of perturbations about the vacuum (2) is non-pathological, i.e. there are neither ghost nor rapid instabilities [16]. The spectrum consist of two tensor modes (graviton polarizations) only, which are, in general, massive. The graviton mass scale is $m \sim \Lambda^{2} / M_{\mathrm{Pl}}$.

During the cosmological evolution, the Universe described by the action (1) is driven to an "attractor" point [18]

$$
\mathcal{F}\left(X, W^{i j}\right) \rightarrow \mathcal{F}\left(Z^{i j}\right) \text { where } Z^{i j}=X^{\gamma} W^{i j},
$$

$\gamma$ being a constant. At the attractor point the theory possesses an additional symmetry,

$$
\phi^{0} \rightarrow \lambda \phi^{0}, \quad \phi^{i} \rightarrow \lambda^{-\gamma} \phi^{i} .
$$


Models described by the action (1) with the function $\mathcal{F}=$ $\mathcal{F}\left(Z^{i j}\right)$ have been studied more intensively [18, 21, 30]. In particular, it has been shown that for $-1<\gamma<0$ and for $\gamma=1$ the cosmological perturbations in these models behave identically to those in GR [21]. For other values of $\gamma$ the behavior of the perturbations may or may not reproduce that of GR depending on the initial conditions.

Another reason to study models characterized by the function $\mathcal{F}=\mathcal{F}\left(Z^{i j}\right)$ comes from the analysis of Newtonian approximation. The gravitational potential of a static source in the model (1), in the linear approximation, has the form [18]

$$
\Phi=M G_{N}\left(-\frac{1}{r}+\mu^{2} r\right)
$$

where $G_{N}=\left(8 \pi \mathrm{M}_{\mathrm{pl}}^{2}\right)^{-1}$ is the Newton's constant and $\mu$ is a constant of order of the graviton mass whose value depends on the particular form of the function $\mathcal{F}$. This constant vanishes at the point where the symmetry (3) holds, that is, where $\mathcal{F}=\mathcal{F}\left(Z^{i j}\right)$. Thus, at the point of the attractor the Newtonian potential remains unmodified.

It has been shown that the standard Schwarzschild metric is a solution to the Einstein equations in massive gravity models possessing the symmetry (3) [27], with the scalar fields given by

$$
\begin{aligned}
\phi^{0} & =\Lambda^{2}\left(t+2 \sqrt{r r_{s}}+r_{s} \ln \frac{\sqrt{r}-\sqrt{r_{s}}}{\sqrt{r}+\sqrt{r_{s}}}\right), \\
\phi^{i} & =\Lambda^{2} x^{i}
\end{aligned}
$$

where $r_{s}$ is the Schwarzschild radius of the black hole. On the other hand, the metric of a rotating black hole is necessarily modified [27] from its standard GR (Kerr) form.

\section{$3 \quad$ Static spherically symmetric ansatz and equations}

A static spherically symmetric configuration in the massive gravity model (1) can be written in the following form,

$$
\begin{aligned}
\mathrm{d} s^{2} & =\alpha(r) \mathrm{d} t^{2}+2 \delta(r) \mathrm{d} t \mathrm{~d} r-\beta(r) \mathrm{d} r^{2}-\kappa(r) \mathrm{d} \Omega^{2} \\
\phi^{0} & =\Lambda^{2}[t+h(r)] \\
\phi^{i} & =\phi(r) \frac{\Lambda^{2} x^{i}}{r} .
\end{aligned}
$$

This field configuration is invariant under two residual coordinate transformations. The first one is an arbitrary change of the radial coordinate $r \rightarrow r^{\prime}=r^{\prime}(r)$, which allows to set either $\kappa=r^{2}$ or $\phi=r$. The second one consist in redefining the time variable $t \rightarrow t^{\prime}=t+\tau(r)$. This last transformation allows to cancel either $\delta(r)$ or $h(r)$. We choose the conditions $\kappa=r^{2}$ and $\delta=0$. Thus, we get the following ansatz,

$$
\begin{aligned}
\mathrm{d} s^{2} & =\alpha(r) \mathrm{d} t^{2}-\beta(r) \mathrm{d} r^{2}-r^{2}\left(\mathrm{~d} \theta^{2}+\sin ^{2} \theta \mathrm{d} \varphi^{2}\right) \\
\phi^{0} & =\Lambda^{2}[t+h(r)] \\
\phi^{i} & =\phi(r) \frac{\Lambda^{2} x^{i}}{r}
\end{aligned}
$$

As compared to GR, this configuration contains two additional radial functions $h(r)$ and $\phi(r)$.

As has been pointed out in Sect. 2, the rotational invariance of the vacuum (and likewise, of the ansatz (4)) requires that the function $\mathcal{F}$ depends on $W^{i j}$ through three combinations $w_{n}=\operatorname{Tr}\left(W^{n}\right)$. These combinations are expressed in terms of the radial functions and their derivatives as follows,

$$
\begin{array}{ll}
w_{1} & =-\left(f_{1}+2 f_{2}\right), \\
w_{2} & =f_{1}^{2}+2 f_{2}^{2}, \\
w_{3} & =-\left(f_{1}^{3}+2 f_{2}^{3}\right),
\end{array}
$$

where the two functions $f_{1}$ and $f_{2}$ are

$$
f_{1}=\frac{\phi^{\prime 2}}{\alpha \beta X}, \quad f_{2}=\frac{\phi^{2}}{r^{2}},
$$

and

$$
X=\frac{\beta-\alpha h^{\prime 2}}{\alpha \beta} .
$$

In these expressions and in what follows, the prime denotes the derivative with respect to the radial coordinate $r$.

After fixing the ansatz (4), the Einstein equations reduce to the following four equations:

$$
\begin{array}{ll}
\mathcal{G}_{0}^{0}=\frac{1}{\mathrm{M}_{\mathrm{pl}}^{2}} \mathcal{T}_{0}^{0}, & \mathcal{G}_{r}^{r}=\frac{1}{\mathrm{M}_{\mathrm{pl}}^{2}} \mathcal{T}_{r}^{r}, \\
\mathcal{G}_{\theta}^{\theta}=\frac{1}{\mathrm{M}_{\mathrm{pl}}^{2}} \mathcal{T}_{\theta}^{\theta}, & 0=\mathcal{T}_{0}^{r},
\end{array}
$$

where $\mathcal{G}_{\mu \nu}$ is the Einstein tensor and $\mathcal{T}_{\mu}^{\nu}$ is the energymomentum tensor of the four Goldstone fields. The other six equations are identically satisfied. The explicit expressions for the components of $\mathcal{G}_{\mu}^{\nu}$ and $\mathcal{T}_{\mu}^{\nu}$ are given in the Appendix.

Consider first the equation $\mathcal{T}_{0}^{r}=0$. Assuming $h^{\prime} \neq 0$, this equation gives

$$
0=X \mathcal{F}_{X}+f_{1}\left(\mathcal{F}_{1}-2 f_{1} \mathcal{F}_{2}+3 f_{1}^{2} \mathcal{F}_{3}\right)
$$

where $\mathcal{F}_{X} \equiv \partial \mathcal{F} / \partial X$ and $\mathcal{F}_{i} \equiv \partial \mathcal{F} / \partial w_{i}$. Furthermore, the time and radial components of the energy-momentum tensor differ by the quantity proportional to eq. (5). Therefore, when this equation holds one has $\mathcal{T}_{0}^{0}=\mathcal{T}_{r}^{r}$. This implies that $\mathcal{G}_{0}^{0}=\mathcal{G}_{r}^{r}$ or, equivalently,

$$
\alpha(r) \beta(r)=1,
$$

in full analogy with the Schwarzschild solution in GR. Hence, the Einstein equations reduce to the following four equations,

$$
\begin{aligned}
1= & \alpha \beta, \\
0= & \frac{\alpha^{\prime}}{r}+\frac{\alpha-1}{r^{2}}-\frac{m^{2}}{2}\left(\mathcal{F}-2 X \mathcal{F}_{X}\right), \\
0= & \frac{\alpha^{\prime}}{r}+\frac{\alpha^{\prime \prime}}{2}-\frac{m^{2}}{2}\left[\mathcal{F}+X \mathcal{F}_{X}\right. \\
& \left.-w_{1} \mathcal{F}_{1}-2 w_{2} \mathcal{F}_{2}-3 w_{3} \mathcal{F}_{3}\right], \\
0= & X \mathcal{F}_{X}+f_{1}\left(\mathcal{F}_{1}-2 f_{1} \mathcal{F}_{2}+3 f_{1}^{2} \mathcal{F}_{3}\right),
\end{aligned}
$$


where $m^{2}=\Lambda^{4} / \mathrm{M}_{\mathrm{pl}}^{2}$. For a generic function $\mathcal{F}$, this system of equations is well defined. Indeed, since the function $h(r)$ enters the equations (6-9) only through the variable $X$, one may consider $X$ as an independent variable instead of $h(r)$. Then the fourth equation allows to find $\phi$ in terms of $X$, while the first equation gives $\beta$ in terms of $\alpha$. The second equation then gives $X$ in terms of $\alpha$ and the third equation allows to determine $\alpha$ as a function of $r$.

\section{Analytical example}

Finding analytical solutions of the non-linear system of equations like (6-9) is impossible for a generic function $\mathcal{F}$. So, in order to get some insight into the behavior of the solutions, let us choose the function $\mathcal{F}$ in such a way that the resulting equations are solvable analytically.

Consider the function $\mathcal{F}$ of the following form,

$$
\begin{aligned}
\mathcal{F}= & c_{0}\left(\frac{1}{X}+w_{1}\right) \\
& +c_{1}\left(w_{1}^{3}-3 w_{1} w_{2}-6 w_{1}+2 w_{3}-12\right),
\end{aligned}
$$

where $c_{0}$ is an arbitrary dimensionless constant and $c_{1}= \pm 1$ (the numerical value of $c_{1}$ can be absorbed into the constant $\Lambda)$. The coefficients inside the parentheses are chosen in such a way that the vacuum (2) is the solution to the Einstein equations at $a=b=1$. Our example contains, therefore, a single continuous free parameter $c_{0}$. Two additional constraints should be imposed on $c_{0}$. The first one comes from the requirement that the graviton is non-tachyonic. This translates into the inequality

$$
c_{0}-6 c_{1} \geq 0 .
$$

The second condition is necessary to ensure that scalar modes with pathological behavior do not reappear upon addition of higher-derivative terms (recall that the model (1) is understood as the low-energy effective theory, so such terms are generically present). This imposes certain constraint on the function $\mathcal{F}$ and its derivatives (cf. eq. (70) of Ref.[16]), which in the case at hand implies

$$
c_{0}>0 \text {. }
$$

We should stress that the choice of the functional form of $\mathcal{F}$ is by no means unique. The particular form (10) has been chosen in order to simplify the solution of the field equations, as will become clear below.

\subsection{The static spherically symmetric solution}

Let us start with eq. (9) which takes the form

$$
0=\frac{1}{X}\left[-c_{0}+\phi^{\prime 2}\left(6 c_{1} \frac{\phi^{4}}{r^{4}}+c_{0}-6 c_{1}\right)\right] .
$$

Because of our choice of the function $\mathcal{F}$, this equation contains the variable $X$ as an overall factor only. Hence, it reduces to a closed differential equation for $\phi$. The solution to this equation is

$$
\phi=b r,
$$

where the constant $b$ satisfies the equation

$$
0=\left(b^{2}-1\right)\left(6 b^{4}+6 b^{2}+c_{0} / c_{1}\right) .
$$

We are interested in real positive values of $b$ (the case $b<0$ can be reduced to $b>0$ by the inversion of coordinates). If $c_{0} / c_{1}>0$ there is only one such solution,

$$
b=1 .
$$

If $c_{0} / c_{1}<0$ there exists another one,

$$
b=\frac{1}{\sqrt{2}}\left(-1+\sqrt{1-2 c_{0} / 3 c_{1}}\right)^{1 / 2} .
$$

Thus, at negative $c_{0} / c_{1}$ we have two different branches of solutions.

The remaining equations (7) and (8) can be written as follows,

$$
\begin{aligned}
& 0=\frac{\alpha^{\prime}}{r}+\frac{\alpha-1}{r^{2}}-3 \Lambda_{c}+m^{2} c_{0}\left(1-\frac{1}{X}\right), \\
& 0=\alpha^{\prime \prime}+\lambda \frac{\alpha-1}{r^{2}}+\left(\frac{\alpha^{\prime}}{r}-3 \Lambda_{c}\right)(2+\lambda),
\end{aligned}
$$

where

$$
\begin{aligned}
\lambda & =-12 b^{6} \frac{c_{1}}{c_{0}}, \\
\Lambda_{c} & =2 m^{2} c_{1}\left(b^{6}-1\right) .
\end{aligned}
$$

Recall that, according to our normalization, the constant $c_{1}$ only takes two values, $c_{1}= \pm 1$.

Eq. (14) is a linear inhomogeneous equation for $\alpha$. Its general solution can be found analytically. Making use of this solution and integrating the remaining equations one finally obtains:

$$
\begin{aligned}
& \alpha(r)=1-\frac{r_{s}}{r}-\frac{S}{r^{\lambda}}+\Lambda_{c} r^{2}, \\
& \beta(r)=1 / \alpha(r), \\
& h(r)= \pm \int \frac{\mathrm{d} r}{\alpha}\left[1-\alpha\left(\frac{S}{c_{0} m^{2}} \frac{\lambda-1}{r^{\lambda+2}}+1\right)^{-1}\right]^{1 / 2}, \\
& \phi(r)=b r .
\end{aligned}
$$

Here $r_{s}$ and $S$ are two integration constants: $r_{s}$ is the Schwarzschild radius, while $S$ is a scalar charge whose presence reflects the modification of the gravitational interaction as compared to GR. At $S=0$ the solution (15) reduces to the conventional Schwarzschild solution describing a black hole of the mass $M=r_{s} /\left(2 G_{N}\right)$.

The behavior of the metric at $r \rightarrow \infty$ depends on the constants $c_{0}$ and $c_{1}= \pm 1$. If $c_{1}=1$, we must also take $c_{0} \geq 6$, and the only solution of eq. (13) in this case is $b=1$. Hence, we have $\Lambda_{c}=0, \lambda<0$, and the metric is growing at infinity as $S r^{|\lambda|}$. Such solutions do not describe asymptotically flat space.

If $c_{1}=-1$, we must take $c_{0}>0$ in order to satisfy eqs. (11) and (12). In this case $\lambda>0$. Two branches of solutions exist, one with zero, and one with non-zero cosmological constant, which can be positive or negative depending on the numerical value of $c_{0}$. In what follows we consider the branch with $\Lambda_{c}=0(b=1)$. 
The behavior of the solutions is determined by the two integration constants $M$ and $S$, and the value of the parameter $\lambda$. If $\lambda<1$, the third term on the r.h.s. of eq. (15) dominates at large distances. The ADM mass of these solutions is infinite. We do not discuss them further here.

If $\lambda>1$, the standard Schwarzschild term dominates at infinity. The ADM mass of such solutions is equal to $M$. The solutions with positive (negative) $M$ have attractive (repulsive) behavior at infinity.

At the origin $r=0$ both terms proportional to $M$ and $S$ are singular, so the metric always possesses a singularity unless $M=S=0$. This singularity may or may not be hidden by the horizon depending on the signs and values of $M$ and $S$. The solutions possessing the horizon are candidates for modified black holes.

The horizon is always present if both $M$ and $S$ are positive. Such black holes have attractive gravitational potential at all distances, which is stronger than for a conventional black hole of the mass $M$. The horizon size of the modified black hole is larger than $r_{s}=2 G_{N} M$.

If $M>0$ and $S<0$, the presence of the horizon depends on the relative values of $S$ and $M$. It exists for sufficiently small $S$. Defining, on dimensional grounds, the mass parameter $s$ associated with $S$ by the relation $|S|=s^{-\lambda}$, the existence of horizon requires that

$$
s M \geq \frac{\lambda}{2 G_{N}}\left(\frac{1}{\lambda-1}\right)^{\frac{\lambda-1}{\lambda}} .
$$

The Newtonian potentials for solutions satisfying and not satisfying the condition (16) are shown in Fig. 1-(a) and (b), respectively. When the horizon exists, the gravitational field is attractive all the way to the horizon. The attraction is weaker than in the case of the usual Schwarzschild black hole of mass $M$, and the horizon size is smaller. The behavior of the gravitational force with distance mimics that of the smaller-mass black hole plus a continuous distribution of "dark matter", with the total mass enclosed within the radius $r$ approaching $M$ as $r \rightarrow \infty$.

Finally, at $M<0$ and $S>0$ the modified black hole anti-gravitates at large distances and gravitates close to the horizon. The attraction changes to repulsion at

$$
r=r_{*} \equiv\left|\frac{\lambda S}{r_{s}}\right|^{\frac{1}{\lambda-1}} .
$$

The corresponding Newtonian potential is shown in Fig. 1(c).

A remark is in order at this point. In the conventional general relativity the constant $r_{s}$ or equivalently, the black hole mass $M$, is also a free parameter which can, in principle, be positive or negative. In GR, however, only positive values make sense for the following reasons. First, negativemass Schwarzschild solutions possess naked singularity at the origin, which is physically unacceptable. Second, the conventional matter satisfies the null energy condition which ensures that any compact spherically-symmetric matter distribution has a positive mass [31]. None of these arguments go through in the case of massive gravity. Fig. 1-(c) gives an example of solution with repulsive behavior at large distances and without naked singularity: as for a conventional black hole, the singularity of this solution is hidden behind the horizon. The positivity of energy is also not expected in massive gravity. This is related to the fact that the background (2) breaks time translations, and only the combination of the time translations with the shifts of $\phi^{0}$ by a constant remains unbroken. In this respect the massive gravity model is exactly analogous to the ghost condensate model [15], where the negative-energy states have been constructed explicitely [32].

\subsection{Correspondence with linear analysis}

The solutions found in the previous section have the asymptotic behavior different from that obtained in the linear perturbation theory in Ref. [20]. In order to compare our results with those of Ref. [20], let us discuss them in the gauge where $h(r)=0, \delta(r) \neq 0$ (cf. Sect. 3). In this gauge the perturbation theory of Ref. [20] corresponds to assuming that the variations of all the metric components are of the same order. In other words, they are formally assigned a small parameter $\epsilon$ to the first power. The solutions described in Ref. [20] satisfy the Einstein equations expanded to the linear order in $\epsilon$.

The solution (15) is not of this type. Transforming it into the gauge $h(r)=0$ one finds that $g_{t r}=\delta(r)$ does not decay as fast as the perturbations of other components, for instance, as $\alpha-1$. In fact, in the equations expanded in powers of perturbations the terms of order $\delta^{2}$ balance those linear in $\alpha-1$. In other words, in the formal expansion of the solution of Sect. 4 in powers of the small parameter $\epsilon$ the perturbation $\delta$ should be assigned the order $\sqrt{\epsilon}$ rather than $\epsilon$. Hence, the solution (15) is non-linear even at large distances from the center. A similar phenomenon has been observed in the context of bi-gravity models in Ref. [28].

Another difference between the solution (15) and the solution to the linearized equations of Ref. [20] is that the former is static, while in the latter only metric components are static (in the gauge $g_{0 i}=0$ ). The scalar fields have time dependence which may be viewed as an accretion of a fluid with zero energy-momentum tensor.

\subsection{Gravitational field of a star}

(See the erratum at the end of this paper.)

In general relativity, one may relate the mass of a star to an integration constant of the vacuum solution in the exterior space by matching the interior and exterior solutions at the star surface (see, e.g., Ref. [33]). In massive gravity, one may try to use the same approach to determine the scalar charge of an ordinary star. The analytical solution in the interior region is required for the matching procedure.

The star is described, to a good approximation, by a diagonal energy-momentum tensor $t_{\mu}^{\nu}=(\rho,-p,-p,-p)$, where $\rho$ and $p$ are the energy density and pressure inside the star, respectively. This energy-momentum tensor is assumed to be responsible for the external gravitational field described by eq. (15). Since there is no direct coupling between the 


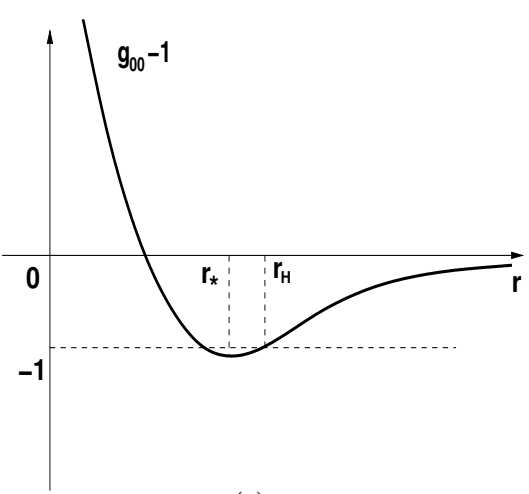

(a)

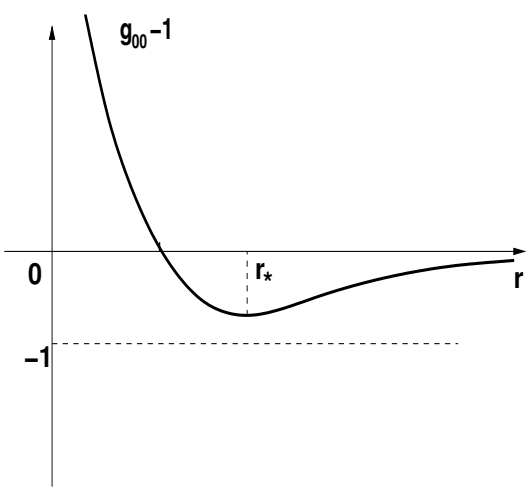

(b)

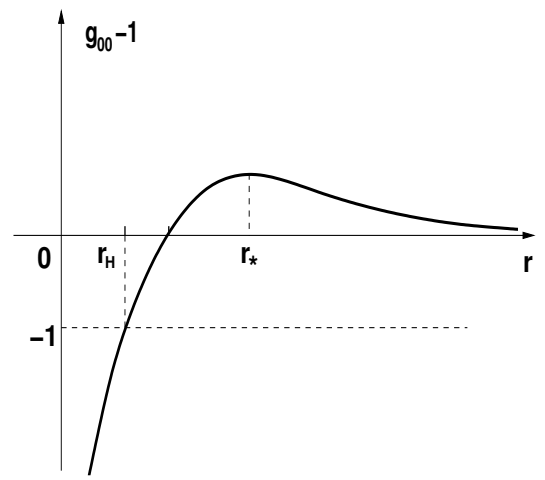

(c)

Figure 1: The deviation of $g_{00}$ from one (proportional to the Newtonian potential $\left.\Phi=\left(g_{00}-1\right) / 2\right)$ for tree different choices of the integration constants. Figs. (a) and (b) correspond to $M>0$ and $S<0$ with the numerical values satisfying (a) and not satisfying (b) eq. (16). Fig. (c) represents solution with $M<0$ and $S>0$.

ordinary matter and the Goldstone fields, $t_{\mu}^{\nu}$ must be conserved separately, $\nabla^{\mu} t_{\mu}^{\nu}=0$. For simplicity, we take the energy density to be constant at $r<R$, where $r=R$ is the surface of the star, and zero outside. The pressure $p$ cannot be chosen independently; it is determined by the conservation of $t_{\mu}^{\nu}$.

Because of the spherical symmetry, the ansatz (4) holds. The Einstein equations in the interior of the star are obtained from eqs. (6-8) by adding the contributions of the energy-momentum of the star, while eq. (9) remains unchanged. The resulting set of equations can be solved analytically. The solution reads

$$
\begin{aligned}
\alpha(r)= & 1-\frac{s_{1}}{r}-\frac{s_{2}}{r^{\lambda}}+\Lambda_{c} r^{2} \\
& +\frac{\rho}{\mathrm{M}_{\mathrm{pl}}^{2}}\left(\frac{r^{2}}{6}-\frac{R^{2}}{2}\right)+\mathcal{O}\left(\rho^{2}\right), \\
\beta(r)= & {\left[1-\frac{s_{1}}{r}-\frac{s_{2}}{r^{\lambda}}+\Lambda_{c} r^{2}-\frac{r^{2} \rho}{3 \mathrm{M}_{\mathrm{pl}}^{2}}\right]^{-1}, } \\
h(r)= & \pm \int \frac{\mathrm{d} r}{\alpha}\left[1-\alpha\left(\frac{s_{2}}{c_{0} m^{2}} \frac{\lambda-1}{r^{\lambda+2}}+1\right)^{-1}\right]^{1 / 2}, \\
\phi(r)= & b r .
\end{aligned}
$$

For simplicity, we have expanded the first equation in powers of $\rho$, while the other relations are exact. Since the geometry inside the star is regular, the integration constants $s_{1}$ and $s_{2}$ must be set to zero.

The interior solution has to be matched with the solution (15) at $r=R$. It is convenient to match the variable $X$ which equals 1 in the interior region. In the gauge $h(r)=0$ this variable is nothing but the $g^{00}$ component of the metric. Hence, it must be continuous. Making use of eqs. (15) one can see that the continuity of $X$ at $r=R$ requires that $S=0$. Therefore, the scalar charge of an ordinary star is zero.

It remains an open question how objects (e.g., black holes) with $S \neq 0$ can be created. The argument given above does not apply to time-dependent configurations, so it is possible that a non-zero scalar charge may be acquired during the gravitational collapse.

\section{$5 \quad \mathcal{F}\left(Z^{i j}\right)$ models}

As mentioned earlier, models characterized by the function $\mathcal{F}$ of a single variable $Z^{i j}=X^{\gamma} W^{i j}$ are of a particular interest. We discuss in this section the exact static spherically symmetric solutions in these models. Our goal is to demonstrate that the solutions found earlier are not specific to the particular form of the action (10) and exist also in the models obeying the symmetry (3).

In Sect. 4 the analytical solutions of eqs. (6-9) were obtained by choosing the function $\mathcal{F}$ in such a way that the dependence on $X$ factors out in eq. (9). Since now $\mathcal{F}$ has only one argument, the derivatives of $\mathcal{F}$ with respect to $X$ and $W^{i j}$ are no more independent. For this reason we did not succeed in constructing non-trivial examples where the Einstein equations are solvable analytically. Hence, to demonstrate the existence of solutions we have to use numerical methods.

Consider the following function $\mathcal{F}$,

$$
\begin{aligned}
\mathcal{F}= & c_{0}\left(z_{1}+2+\frac{z_{1}^{3}-6 z_{1} z_{2}+8 z_{3}}{3}\right) \\
& +2 c_{1}\left(z_{1}^{2}-2 z_{2}-1-2 \frac{z_{1}^{3}-6 z_{1} z_{2}+8 z_{3}}{3}\right),
\end{aligned}
$$

were $z_{n}=\operatorname{Tr}\left(Z^{n}\right)$ are tree independent scalars made out of $Z^{i j}, z_{n}=\left(X^{\gamma}\right)^{n} w_{n}$. The coefficients in front of individual terms have been adjusted so that the flat metric and the scalar fields given by eq. (2) solve the field equations at $a=b=1$. For the vacuum configuration (2) one has $Z^{i j}=$ $-\delta^{i j}$. We are interested in solutions to the field equations that asymptote to this vacuum state.

In addition to the adjustments already made, the following inequality should be imposed on the coefficients $c_{0}$ and $c_{1}$ to ensure that the graviton is non-tachyonic,

$$
c_{0}-2 c_{1} \geq 0 .
$$

This guarantees that the square of the graviton mass is nonnegative. Moreover, this inequality is sufficient for the absence of pathological scalar modes which may appear upon 


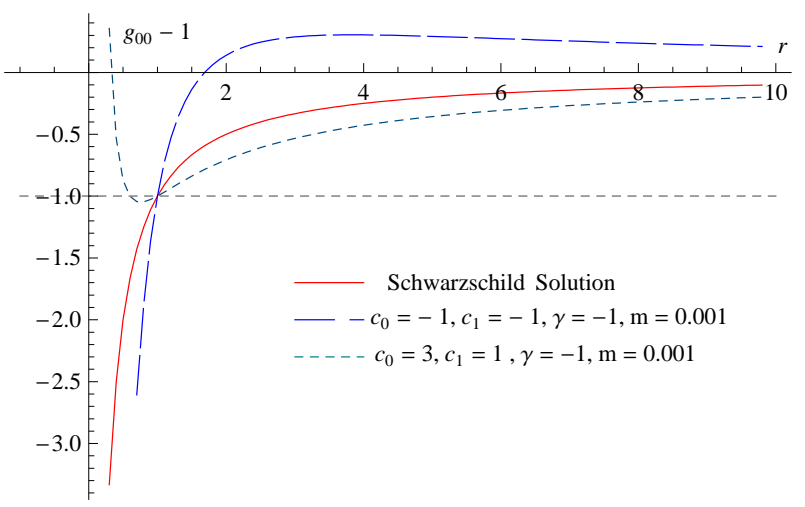

Figure 2: The deviation of $g_{00}$ from one for three different cases: the usual Schwarzschild solution (solid line) and two solutions corresponding to different values of the parameters of the function (17) (long-dashed and short-dashed lines). The integration constants of these solutions have been chosen such that the external horizon is located at $r=1$.

addition of higher-derivative terms. As in the previous example, the overall scale of the coefficients $c_{0}$ and $c_{1}$ can be absorbed in the parameter $\Lambda$, so without loss of generality we may set $c_{1}= \pm 1$.

For this class of models, the field equations (6-9) may be viewed as equations for $\alpha(r), \beta(r), \xi(r) \equiv X^{\gamma} f_{1}-1$ and $\psi(r) \equiv X^{\gamma} f_{2}-1$. Then eq. (6) gives $\beta$ in terms of $\alpha$, while eq. (9) enables to express $\psi$ in terms of $\xi$. The two remaining equations form a coupled set of non-linear equations for $\alpha$ and $\xi$; they have to be solved numerically.

The numerical solutions are shown in Fig. 2 for different value of the parameters $c_{0}$ and $c_{1}$. For all these graphs, we have assumed that the external horizon is located at $r=1$ and that $\xi=100$ at the horizon. The large value of $\xi$ is chosen in order to make the difference between the modified solution and the Schwarzschild solution visible on the plot (large values of $\xi$ correspond to large scalar charge $S$ of the previous section).

The plots show the behavior qualitatively similar to that discussed in Section 4. In particular:

- if $c_{0}=c_{1}=-1$, the Newtonian potential $2 \Phi=\alpha-1$ is attractive at short distances and becomes repulsive at larger distances;

- if $c_{0}=3$ and $c_{1}=1$, the Newtonian potential is attractive outside of the horizon, and becomes repulsive close to the singularity.

The deviations from the Schwarzschild metric are larger for larger values of the "scalar charge" (parameterized by the value of $\xi$ at the horizon). The Schwarzschild solution is recovered at $\xi \rightarrow 0$.

\section{Discussion}

To summarize, there exist spherically symmetric vacuum solutions in massive gravity models which depend on two integration constants, the mass $M$ and an extra parameter
$S$ which can be called the "scalar charge". At zero value of the scalar charge the standard Schwarzschild solution is recovered, while at non-zero $S$ the metric is modified with respect to the Schwarzschild case.

The solutions having non-zero scalar charge exhibit much reacher behavior than the Schwarzschild solution in GR. As can be seen from the explicit example of Sect. 4 , both the short and long distance behavior may be modified depending on the parameters of the model.

Unlike in General Relativity, the solutions may have a negative ADM mass. Such solutions have repulsive gravitational interaction at large distances. At short distances the repulsion may change to attraction and give rise to the horizon, hiding the singularity at the origin. Such solutions represent anti-gravitating black holes.

In the case of a positive ADM mass, the $S$-dependent contributions may make the gravitational attraction weaker at short distances (cf. fig. 1-(a)). In this case the gravitational force decays with distance slower than $1 / r^{2}$, thus mimicking the presence of dark matter. Interestingly, solutions with the same value of $M$ but different scalar charge $S$ have different behavior, which corresponds to different amount of the apparent "dark matter". This is in contrast with other models possessing modifications of the gravitational potential $[4,6,10]$, where the modification of the gravitational force is determined by the parameters of the model.

It is currently an open question how objects with nonzero scalar charge may be created. As has been argued in Sect. 4.3, the absence of direct coupling between the Goldstone fields and ordinary matter results in zero scalar charges of static matter distributions. Thus, the gravitational field of ordinary stars is described by the $S=0$ solutions, i.e., by the standard Schwarzschild metric. This may be not the case for black holes, especially the super-massive black holes in the centers of galaxies, which may be of primordial origin $[34,35]$. In any case, this question requires further investigation.

Another open question is the stability of the modified black hole solutions. Several kinds of instabilities may be present. Among perturbations of the solutions there may exist unstable modes with the characteristic time scale of order of the horizon size; in this case the interpretation in terms of black holes is not possible. Second potential source of problems is generic presence of the higher-derivative terms not included in the action (1). One has to check that the 2-parameter family of modified black holes survives their inclusion. By analogy with the ghost condensate case, one may expect that these terms produce at least a slow Jeanstype instability [15], which is not, however, dangerous for the black hole interpretation. Finally, the presence of negative mass solutions may lead to instabilities of the quantummechanical nature similar to those found in Ref.[36].

To conclude this list, let us mention also the solutions satisfying $h=0$ which were not considered in this paper. In this case the ten Einstein equations reduce to three equations for $\alpha, \beta$ and $\phi$ which form a (generically) well-defined system. It remains to be seen whether this system has asymptotically flat solutions. In any case, the Schwarzschild solution does not belong to this class which is characterized by $\alpha \beta \neq 1$. 


\section{Acknowledgments}

We are grateful to S. Dubovsky for valuable discussions and comments on the manuscript. The work of M.B. is supported by the Belgian Fond pour la Formation à la Recherche dans l'Industrie et dans l'Agriculture (FRIA). The work of P.T. is supported by IISN, Belgian Science Policy (under contract IAP V/27).

\section{Appendix}

For the ansatz (4) discussed in Section 3, the non-zero components of the Einstein tensor are given by the following relations

$$
\begin{aligned}
\mathcal{G}_{0}^{0} & =\frac{1}{r^{2}}\left[1-\left(\frac{r}{\beta}\right)^{\prime}\right] \\
\mathcal{G}_{r}^{r} & =\frac{1}{r^{2}}\left(1-\frac{\alpha+r \alpha^{\prime}}{\alpha \beta}\right), \\
\mathcal{G}_{\theta}^{\theta} & =\mathcal{G}_{\varphi}^{\varphi}=-\frac{1}{4 r}\left[\frac{\alpha^{\prime}+r \alpha^{\prime \prime}}{\alpha \beta}+\left(\frac{2 \alpha+r \alpha^{\prime}}{\alpha \beta}\right)^{\prime}\right] .
\end{aligned}
$$

The energy-momentum tensor of the Goldstone fields is expressed through

$$
\begin{aligned}
\mathcal{T}_{\mu}^{\nu}= & -\frac{1}{2} \Lambda^{4} \delta_{\mu}^{\nu} \mathcal{F}+\mathcal{F}_{X} \partial^{\nu} \phi^{0} \partial_{\mu} \phi^{0} \\
& +\frac{\partial \mathcal{F}}{\partial W^{i j}}\left[\partial^{\nu} \phi^{i} \partial_{\mu} \phi^{j}+\frac{V^{i} V^{j}}{X^{2}} \partial^{\nu} \phi^{0} \partial_{\mu} \phi^{0}\right. \\
& \left.-\frac{V^{j}}{X}\left(\partial^{\nu} \phi^{i} \partial_{\mu} \phi^{0}+\partial^{\nu} \phi^{0} \partial_{\mu} \phi^{i}\right)\right]
\end{aligned}
$$

where $\mathcal{F}_{X} \equiv \partial \mathcal{F} / \partial X$ and

$$
V^{i}=\frac{\partial^{\mu} \phi^{0} \partial_{\mu} \phi^{i}}{\Lambda^{4}}
$$

For functions $\mathcal{F}$ which are invariant under rotations of the Goldstone fields $\phi^{i}$ internal space, the derivatives of $\mathcal{F}$ with respect to $W^{i j}$ are given by

$$
\frac{\partial \mathcal{F}}{\partial W^{i j}}=\mathcal{F}_{1} \delta_{i j}+2 \mathcal{F}_{2} W^{i j}+3 \mathcal{F}_{3} W^{i k} W^{k j},
$$

where $\mathcal{F}_{i} \equiv \partial \mathcal{F} / \partial w_{i}$. Therefore, the components of the energy-momentum tensor which are not identically zero are given by

$$
\begin{aligned}
\mathcal{T}_{0}^{0}= & \Lambda^{4}\left[-\frac{1}{2} \mathcal{F}+\frac{1}{\alpha}\left(\mathcal{F}_{X}+\frac{\partial \mathcal{F}}{\partial W^{i j}} \frac{V^{i} V^{j}}{X^{2}}\right)\right], \\
\mathcal{T}_{r}^{r}= & \Lambda^{4}\left[-\frac{1}{2} \mathcal{F}-\left(\mathcal{F}_{X}+\frac{\partial \mathcal{F}}{\partial W^{i j}} \frac{V^{i} V^{j}}{X^{2}}\right) \frac{h^{\prime 2}}{\beta}\right] \\
& +\frac{1}{\beta} \frac{\partial \mathcal{F}}{\partial W^{i j}}\left(-\partial_{r} \phi^{i} \partial_{r} \phi^{j}+\frac{2 V^{j}}{X} \partial_{r} \phi^{i} \partial_{r} \phi^{0}\right), \\
\mathcal{T}_{\theta}^{\theta}= & \mathcal{T}_{\varphi}^{\varphi}=-\frac{1}{2} \Lambda^{4} \mathcal{F}+\frac{\partial \mathcal{F}}{\partial W^{i j}} \partial^{\theta} \phi^{i} \partial_{\theta} \phi^{j}, \\
\mathcal{T}_{0}^{r}= & -\frac{\Lambda^{4} h^{\prime}}{\beta}\left[\mathcal{F}_{X}+\frac{\partial \mathcal{F}}{\partial W^{i j}}\left(\frac{V^{i} V^{j}}{X^{2}}+\frac{\partial_{r} \phi^{i} \partial_{r} \phi^{j}}{\beta \Lambda^{4} X}\right)\right] .
\end{aligned}
$$

\section{Erratum}

In the original version of this paper, we derived modified black hole solutions in a certain type of scalar-tensor gravity models. Unlike in General Relativity (GR), these solutions depend on two parameters (integration constants): the mass $M$ and an additional parameter called the scalar charge $S$. In Sect. 4.3, in a particular subclass of the massive gravity models, we discussed the possibility for an ordinary star to have a non zero scalar charge. This question is important for understanding mechanisms by which black holes with non-zero $S$ may be formed. Non-zero scalar charge of massive bodies may also result in additional constraints on the graviton mass [37].

By solving the Einstein equations in the interior and exterior regions of a star and matching the two solutions, we came to the (erroneous) conclusion that the scalar charge of the stars must be zero. The authors of Ref. [37] have obtained a different result. Revising our calculations, we found a mistake in the solution presented in Sect. 4.3 (this solution corresponds to the interior part of the star). Thus, our conclusion about the zero scalar charge of ordinary stars in those models is wrong. This error changes neither the validity of the modified black hole solutions themselves, nor other conclusions of the paper.

We give below the correct expressions for the scalar charges and corresponding corrections to the black hole mass for the models considered in this paper. They are most easily obtained by using the quasi-linear equations of Ref. [38].

For the models discussed in Sect. 4, the Newtonian potential in the week field limit $\Phi$ reads

$$
\begin{aligned}
\Phi= & -G\left(\frac{M}{r}+\frac{S}{r^{\lambda}}\right) \theta(r-R) \\
& +\frac{G M_{0}}{2 R}\left[\frac{r^{2}}{R^{2}}-3+3 \lambda m_{2}^{2} R^{2}\left(\mu_{0}\left(\frac{r}{R}\right)^{\lambda+1}\right.\right. \\
& \left.\left.+\mu_{1}\left(\frac{r}{R}\right)^{4}+\mu_{2}\left(\frac{r}{R}\right)^{2}\right)\right] \theta(R-r) .
\end{aligned}
$$

Here $m_{2}^{2} \propto \Lambda^{4} / \mathrm{M}_{\mathrm{pl}}^{2}$ is the graviton mass, the parameter $\lambda$ is defined in Sect. 4.1 and the parameters $\mu_{0}, \mu_{1}$ and $\mu_{2}$ are some combinations of $\lambda$ of order one. The mass $M$ and the scalar charge $S$ of a star are given by

$$
\begin{aligned}
M & =M_{0}\left(1+\frac{3 m_{2}^{2} R^{2} \lambda}{10(\lambda-1)(2+\lambda)}\right), \\
S & =\frac{9 \lambda M_{0} m_{2}^{2} R^{1+\lambda}}{2(1-\lambda)(2+\lambda)(4+\lambda)(1+2 \lambda)},
\end{aligned}
$$

where $M_{0}=4 \pi R^{3} \varrho / 3$ is the rest mass of the star. The Newtonian potential of an ordinary star acquires two new contributions as compared to its value in GR, which are in agreement with Ref. [37].

This solution has an interesting asymptotical behavior for $\lambda>1$ : at very large distances from the source $R / r \rightarrow 0$, the potential $\Phi$ reduces to the usual solution of GR. Hence, the potential of a point source is simply given by its value in GR, in agreement with the results of Ref. [38] which studied the same equations in the case of point sources. 
For the class of models considered in Sect. 5 the situation is different. In this case the Newtonian potential of a star in the week field limit reads

$$
\Phi=-\frac{G M_{0}}{r} \theta(r-R)+\frac{G M_{0}}{2 R}\left(\frac{r^{2}}{R^{2}}-3\right) \theta(R-r),
$$

so that no extra terms are present and the potential is the same as in GR. The scalar charge of the star $S$ and the correction to the star mass are both zero. Correspondingly, no extra constraints on the graviton mass arise in these models.

\section{References}

[1] Supernova Cosmology Project, S. Perlmutter et al., Astrophys. J. 517, 565 (1999), astro-ph/9812133.

[2] WMAP, J. Dunkley et al., Astrophys. J. Suppl. 180, 306 (2009), 0803.0586.

[3] SDSS, J. K. Adelman-McCarthy, Astrophys. J. Suppl. 172, 634 (2007), 0707.3380.

[4] M. Milgrom, Astrophys. J. 270, 371 (1983).

[5] J. D. Bekenstein, Phys. Rev. D70, 083509 (2004), astro$\mathrm{ph} / 0403694$.

[6] G. R. Dvali, G. Gabadadze, and M. Porrati, Phys. Lett. B485, 208 (2000), hep-th/0005016.

[7] R. Gregory, V. A. Rubakov, and S. M. Sibiryakov, Phys. Rev. Lett. 84, 5928 (2000), hep-th/0002072.

[8] T. Damour and I. I. Kogan, Phys. Rev. D66, 104024 (2002), hep-th/0206042.

[9] D. Blas, C. Deffayet, and J. Garriga, Phys. Rev. D76, 104036 (2007), 0705.1982.

[10] S. M. Carroll, V. Duvvuri, M. Trodden, and M. S. Turner, Phys. Rev. D70, 043528 (2004), astro-ph/0306438.

[11] G. Dvali, O. Pujolas, and M. Redi, Phys. Rev. Lett. 101, 171303 (2008), 0806.3762.

[12] G. Gabadadze, (2003), hep-ph/0308112.

[13] V. A. Rubakov and P. G. Tinyakov, Phys. Usp. 51, 759 (2008), 0802.4379 .

[14] T. P. Sotiriou and V. Faraoni, (2008), 0805.1726.

[15] N. Arkani-Hamed, H.-C. Cheng, M. A. Luty, and S. Mukohyama, JHEP 05, 074 (2004), hep-th/0312099.

[16] S. L. Dubovsky, JHEP 10, 076 (2004), hep-th/0409124.

[17] V. A. Rubakov, (2004), hep-th/0407104.

[18] S. L. Dubovsky, P. G. Tinyakov, and I. I. Tkachev, Phys. Rev. D72, 084011 (2005), hep-th/0504067.

[19] J. H. Taylor and J. M. Weisberg, Astrophys. J. 253, 908 (1982).

[20] S. L. Dubovsky, P. G. Tinyakov, and I. I. Tkachev, Phys. Rev. Lett. 94, 181102 (2005), hep-th/0411158.

[21] M. V. Bebronne and P. G. Tinyakov, Phys. Rev. D76, 084011 (2007), 0705.1301.

[22] McClintock, J. E. and Remillard, R. A., Astrophys. J. 308, 110 (1986).

[23] J. E. McClintock and R. A. Remillard, (2003), astro-ph/0306213.

[24] R. Genzel, A. Eckart, T. Ott, and F. Eisenhauer, Mon. Not. Roy. Astron. Soc. 291, 219 (1997).

[25] S. Gillessen et al., (2008), 0810.4674.

[26] C. M. Will, Living Rev. Rel. 9, 3 (2005), gr-qc/0510072.

[27] S. Dubovsky, P. Tinyakov, and M. Zaldarriaga, JHEP 11, 083 (2007), 0706.0288.

[28] Z. Berezhiani, D. Comelli, F. Nesti, and L. Pilo, JHEP 07, 130 (2008), 0803.1687.
[29] R. L. Arnowitt, S. Deser, and C. W. Misner, Phys. Rev. 122, 997 (1961).

[30] M. V. Bebronne, Phys. Lett. B668, 432 (2008), 0806.1167.

[31] S. W. Hawking and G. F. R. Ellis, The Large scale structure of space-time, Cambridge University Press, Cambridge (1973).

[32] N. Arkani-Hamed, H.-C. Cheng, M. A. Luty, S. Mukohyama, and T. Wiseman, JHEP 01, 036 (2007), hep-ph/0507120.

[33] S. Weinberg, Gravitation and Cosmology: Principles and Applications of the General Theory of Relativity (Wiley, 1972).

[34] B. J. Carr and S. W. Hawking, Mon. Not. Roy. Astron. Soc. 168, 399 (1974)

[35] B. J. Carr, (2005), astro-ph/0504034.

[36] D. Krotov, C. Rebbi, V. A. Rubakov, and V. Zakharov, Phys. Rev. D71, 045014 (2005), hep-ph/0407081.

[37] D. Comelli, F. Nesti, and L. Pilo, Phys. Rev. D83, 084042 (2011), 1010.4773 .

[38] M. V. Bebronne, Phys. Rev. D82, 024020 (2010), 1005.4547. 


\title{
Black hole solutions in massive gravity
}

\author{
Michael V. Bebronne ${ }^{\mathrm{a}}$, Peter G. Tinyakov ${ }^{\mathrm{a}, \mathrm{b}}$ \\ ${ }^{a}$ Service de Physique Théorique, Université Libre de Bruxelles (ULB), \\ CP225, boulevard du Triomphe, B-1050 Bruxelles, Belgium. \\ ${ }^{b}$ Institute for Nuclear Research of the Russian Academy of Sciences, \\ 60th October Anniversary Prospect, 7a, 117312 Moscow, Russia.
}

\begin{abstract}
The static vacuum spherically symmetric solutions in massive gravity are obtained both analytically and numerically. The solutions depend on two parameters (integration constants): the mass $M$ (or, equivalently, the Schwarzschild radius), and an additional parameter, the "scalar charge" $S$. At zero value of $S$ and positive mass the standard Schwarzschild

- black hole solutions are recovered. Depending on the parameters of the model and the signs of $M$ and $S$, the solutions may or may not have horizon. Those with the horizon describe modified black holes provided they are stable against small perturbations. In the analytically solvable example, the modified black hole solutions may have both attractive and repulsive (anti-gravitating) behavior at large distances. At intermediate distances the gravitational potential of a modified black hole may mimics the presence of dark matter. Modified black hole solutions are also found numerically in more realistic massive gravity models which are attractors of the cosmological evolution.
\end{abstract}

- Key words: Massive gravity, Spherically symmetric solutions, Black holes. ULB reference: ULB-TH/09-05

\section{Introduction}

Recent advances in observational cosmology $[1,2,3]$ have revived interest in alternative theories of gravity in which the gravitational interaction is modified in the infrared domain and which could - potentially - explain the accelerated expansion of the Universe without introducing the dark energy and matter components. Theoretical consistency and existing experimental data impose severe constraints on such models. Different approaches to the problem have been discussed in the literature (see, e.g., Refs. [4, 5, 6, 7, 8, 9, 10, 11], and Refs. [12, 13, 14] for reviews). One of them employs spontaneous breaking of Lorentz symmetry by space-time dependent condensates of scalar fields $[15,16]$ coupled to gravity in a covariant way via a derivative coupling. The resulting theory may have a non-pathological perturbative behavior about the broken vacuum $[17,16]$ and exhibit modifications of gravitational interactions at large scales (see Sect. 2 for more details). In particular, graviton may acquire a non-zero mass, which is the reason to call these models massive gravity models.

Existing experimental data constrain the mass of the graviton and other parameters of massive gravity models. Absence of Lorentz invariance makes the constraints weaker than one would expect in a Lorentz-invariant theory: the Newton's potential remains unmodified in the linear approximation despite non-zero graviton mass [18], so that Solar system constraints are satisfied for rather large masses. The constraint on the mass of the graviton comes from the emission of gravitational waves by binary pulsars which is responsible for their spin-down [19]. Consistency of observations with GR requires the graviton mass to be smaller than the inverse period of orbital motion of the binary system, that is [20]

$$
m \lesssim 10^{-19} \mathrm{eV} .
$$

Standard cosmological solutions may be reproduced in massive gravity [18]. Further constraints on the parameters of the model are imposed by the growth of perturbations and structure formation in the post-inflationary epoch [21].

In General Relativity (GR), a crucial role is played by the spherically symmetric vacuum solution to the Einstein equations - the Schwarzschild solution. This role is twofold: First, this solution describes the metric outside of spherical non-rotating bodies and gives rise, in the weak field limit, to the Newtonian gravity. It provides therefore a useful approximation in many astrophysical situations.

Second, the Schwarzschild solution describes the result of a gravitational collapse, the black hole. Although the existence of black holes has not yet been directly confirmed, there exists an indirect evidence that some of binary stellar systems contain black holes as one of the companion $[22,23]$, and that many galaxies, including the Milky Way, harbor super-massive black holes in their centers $[24,25]$. It is conceivable that black holes will be directly observed in the near future, and that their properties, including the metric configuration near the horizon, will be quantitatively tested [26], thus providing a probe of GR in a fully non-linear regime. 
The Schwarzschild metric, together with properly arranged scalar fields is a solution to Einstein equations in massive gravity as well [27]. However, the properties of black holes are, in general, expected to be different. In particular, rotating black holes are certainly modified, and, more generally, black holes are expected to have hair [27]. The possible existence of black hole hair in massive gravity models suggests that there might exist spherically symmetric solutions other than the Schwarzschild one. In this paper we construct explicit examples of such solutions.

We found a new class of vacuum spherically-symmetric solutions in massive gravity which depend, in addition to the mass $M$ (equivalently, the Schwarzschild radius), on one more parameter which we call the "scalar charge" $S$. At zero value of this parameter the standard Schwarzschild solution is recovered, while at non-zero values of $S$ the Schwarzschild metric gets modified. The modified solution is non-linear at all distances; it cannot be obtained in the linear approximation. Similar phenomenon has been previously found in the context of bi-gravity models [28]. The new solutions may have event horizons and are, therefore, candidates for modified black holes. We found both analytical and numerical examples of such modified black holes.

The analytical solutions found in a particular massive gravity model show a variety of different behaviors. Depending on the parameters of the model, the deviation of the metric from the Minkowski one may decay at infinity as $1 / r$ or slower. The solutions thus may have finite or infinite ADM [29] mass, respectively. In the case of a finite mass, this mass may be positive or negative depending on the sign of $M$. In either case the singularity at the origin may be hidden by the horizon. The solutions with negative mass exhibit an anti-gravitating behavior at large distances from the center.

The modified gravitational potential may decay slower than $1 / r$ in a certain distance range. Such a behavior would mimic the effect of the dark matter. Interestingly, the modification depends not only on the parameters of the model, but also on the scalar charge and the mass of the solution. Thus, the apparent amount of the "dark matter" may be different for objects of the same mass, in contrast with other models of modified gravity.

This paper is organized as follows. In Sect. 2 we briefly review the massive gravity model and summarize previous results about the Newtonian potential in this model. In Sect. 3 we present the spherically symmetric ansatz and reduce the Einstein equations to four ordinary differential equations. In Sect. 4 we choose a particular model and find analytical solutions to the Einstein equations in this model. In Sect. 5 we demonstrate, by numerical computation, that modified black hole solutions exist in more realistic massive gravity models which are attractors of the cosmological evolution. Finally, Sect. 6 contains the summary and discussion of our results.

\section{The massive gravity model}

In this paper we consider the massive gravity model described by the following action [16],

$$
\mathcal{S}=\int \mathrm{d} x^{4} \sqrt{-g}\left[-\mathrm{M}_{\mathrm{pl}}^{2} \mathcal{R}+\mathcal{L}_{\mathrm{m}}+\Lambda^{4} \mathcal{F}\right]
$$

The first two terms are the curvature and the Lagrangian of the minimally coupled ordinary matter; they comprise the standard GR action. The third term describes four scalar fields $\phi^{0}, \phi^{i}$ whose space-time dependent vacuum expectation values break spontaneously the Lorentz symmetry. These fields are minimally coupled to gravity through a derivative coupling; they will be referred to as the Goldstone fields. We consider the functions $\mathcal{F}$ which depend on two particular combinations of the derivatives of the Goldstone fields, $\mathcal{F}=\mathcal{F}\left(X, W^{i j}\right)$, where

$$
\begin{aligned}
X & =\frac{\partial^{\mu} \phi^{0} \partial_{\mu} \phi^{0}}{\Lambda^{4}}, \\
W^{i j} & =\frac{\partial^{\mu} \phi^{i} \partial_{\mu} \phi^{j}}{\Lambda^{4}}-\frac{\partial^{\mu} \phi^{i} \partial_{\mu} \phi^{0} \partial^{\nu} \phi^{j} \partial_{\nu} \phi^{0}}{\Lambda^{8} X} .
\end{aligned}
$$

The constant $\Lambda$ has the dimension of mass. The model is understood as the low-energy effective theory valid below the scale $\Lambda$.

The vacuum configuration has the form

$$
g_{\mu \nu}=\eta_{\mu \nu}, \quad \phi^{0}=a \Lambda^{2} t, \quad \phi^{i}=b \Lambda^{2} x^{i},
$$

where $a$ and $b$ are two constants determined by the requirement that the energy-momentum tensor associated with the four scalar fields vanishes in the Minkowski background. The configuration (2) is, therefore, a solution to the Einstein equations. The constants $a$ and $b$ may be set to one by the redefinition of fields, which we assume to be the case in what follows.

For functions $\mathcal{F}$ which are invariant under rotations of the Goldstone fields $\phi^{i}$ in the internal space (i.e., those depending on $W^{i j}$ through three combinations $w_{n}=\operatorname{Tr} W^{n}$, $n=1,2,3$ ), the background (2) preserves the rotational symmetry. The Lorentz symmetry is, in general, broken.

The action of the Goldstone fields with functions $\mathcal{F}$ depending only on $X$ and $W^{i j}$ is invariant under the following symmetry,

$$
\phi^{i} \rightarrow \phi^{i}+\chi^{i}\left(\phi^{0}\right)
$$

where $\chi^{i}$ are arbitrary functions of $\phi^{0}$. Because of this symmetry, the behavior of perturbations about the vacuum (2) is non-pathological, i.e. there are neither ghost nor rapid instabilities [16]. The spectrum consist of two tensor modes (graviton polarizations) only, which are, in general, massive. The graviton mass scale is $m \sim \Lambda^{2} / M_{\mathrm{Pl}}$.

During the cosmological evolution, the Universe described by the action (1) is driven to an "attractor" point [18]

$$
\mathcal{F}\left(X, W^{i j}\right) \rightarrow \mathcal{F}\left(Z^{i j}\right) \quad \text { where } \quad Z^{i j}=X^{\gamma} W^{i j},
$$


$\gamma$ being a constant. At the attractor point the theory possesses an additional symmetry,

$$
\phi^{0} \rightarrow \lambda \phi^{0}, \quad \phi^{i} \rightarrow \lambda^{-\gamma} \phi^{i} .
$$

Models described by the action (1) with the function $\mathcal{F}=$ $\mathcal{F}\left(Z^{i j}\right)$ have been studied more intensively [18, 21, 30]. In particular, it has been shown that for $-1<\gamma<0$ and for $\gamma=1$ the cosmological perturbations in these models behave identically to those in GR [21]. For other values of $\gamma$ the behavior of the perturbations may or may not reproduce that of GR depending on the initial conditions.

Another reason to study models characterized by the function $\mathcal{F}=\mathcal{F}\left(Z^{i j}\right)$ comes from the analysis of Newtonian approximation. The gravitational potential of a static source in the model (1), in the linear approximation, has the form [18]

$$
\Phi=M G_{N}\left(-\frac{1}{r}+\mu^{2} r\right)
$$

where $G_{N}=\left(8 \pi \mathrm{M}_{\mathrm{pl}}^{2}\right)^{-1}$ is the Newton's constant and $\mu$ is a constant of order of the graviton mass whose value depends on the particular form of the function $\mathcal{F}$. This constant vanishes at the point where the symmetry (3) holds, that is, where $\mathcal{F}=\mathcal{F}\left(Z^{i j}\right)$. Thus, at the point of the attractor the Newtonian potential remains unmodified.

It has been shown that the standard Schwarzschild metric is a solution to the Einstein equations in massive gravity models possessing the symmetry (3) [27], with the scalar fields given by

$$
\begin{aligned}
\phi^{0} & =\Lambda^{2}\left(t+2 \sqrt{r r_{s}}+r_{s} \ln \frac{\sqrt{r}-\sqrt{r_{s}}}{\sqrt{r}+\sqrt{r_{s}}}\right), \\
\phi^{i} & =\Lambda^{2} x^{i},
\end{aligned}
$$

where $r_{s}$ is the Schwarzschild radius of the black hole. On the other hand, the metric of a rotating black hole is necessarily modified [27] from its standard GR (Kerr) form.

\section{Static spherically symmetric ansatz and equa- tions}

A static spherically symmetric configuration in the massive gravity model (1) can be written in the following form,

$$
\begin{aligned}
\mathrm{d} s^{2} & =\alpha(r) \mathrm{d} t^{2}+2 \delta(r) \mathrm{d} t \mathrm{~d} r-\beta(r) \mathrm{d} r^{2}-\kappa(r) \mathrm{d} \Omega^{2}, \\
\phi^{0} & =\Lambda^{2}[t+h(r)], \\
\phi^{i} & =\phi(r) \frac{\Lambda^{2} x^{i}}{r} .
\end{aligned}
$$

This field configuration is invariant under two residual coordinate transformations. The first one is an arbitrary change of the radial coordinate $r \rightarrow r^{\prime}=r^{\prime}(r)$, which allows to set either $\kappa=r^{2}$ or $\phi=r$. The second one consist in redefining the time variable $t \rightarrow t^{\prime}=t+\tau(r)$. This last transformation allows to cancel either $\delta(r)$ or $h(r)$. We choose the conditions $\kappa=r^{2}$ and $\delta=0$. Thus, we get the following ansatz,

$$
\begin{aligned}
\mathrm{d} s^{2} & =\alpha(r) \mathrm{d} t^{2}-\beta(r) \mathrm{d} r^{2}-r^{2}\left(\mathrm{~d} \theta^{2}+\sin ^{2} \theta \mathrm{d} \varphi^{2}\right), \\
\phi^{0} & =\Lambda^{2}[t+h(r)], \\
\phi^{i} & =\phi(r) \frac{\Lambda^{2} x^{i}}{r} .
\end{aligned}
$$

As compared to GR, this configuration contains two additional radial functions $h(r)$ and $\phi(r)$.

As has been pointed out in Sect. 2, the rotational invariance of the vacuum (and likewise, of the ansatz (4)) requires that the function $\mathcal{F}$ depends on $W^{i j}$ through three combinations $w_{n}=\operatorname{Tr}\left(W^{n}\right)$. These combinations are expressed in terms of the radial functions and their derivatives as follows,

$$
\begin{aligned}
& w_{1}=-\left(f_{1}+2 f_{2}\right), \\
& w_{2}=f_{1}^{2}+2 f_{2}^{2}, \\
& w_{3}=-\left(f_{1}^{3}+2 f_{2}^{3}\right),
\end{aligned}
$$

where the two functions $f_{1}$ and $f_{2}$ are

$$
f_{1}=\frac{\phi^{\prime 2}}{\alpha \beta X}, \quad f_{2}=\frac{\phi^{2}}{r^{2}},
$$

and

$$
X=\frac{\beta-\alpha h^{\prime 2}}{\alpha \beta} .
$$

In these expressions and in what follows, the prime denotes the derivative with respect to the radial coordinate $r$.

After fixing the ansatz (4), the Einstein equations reduce to the following four equations:

$$
\begin{array}{ll}
\mathcal{G}_{0}^{0}=\frac{1}{\mathrm{M}_{\mathrm{pl}}^{2}} \mathcal{T}_{0}^{0}, & \mathcal{G}_{r}^{r}=\frac{1}{\mathrm{M}_{\mathrm{pl}}^{2}} \mathcal{T}_{r}^{r}, \\
\mathcal{G}_{\theta}^{\theta}=\frac{1}{\mathrm{M}_{\mathrm{pl}}^{2}} \mathcal{T}_{\theta}^{\theta}, & 0=\mathcal{T}_{0}^{r},
\end{array}
$$

where $\mathcal{G}_{\mu \nu}$ is the Einstein tensor and $\mathcal{T}_{\mu}^{\nu}$ is the energymomentum tensor of the four Goldstone fields. The other six equations are identically satisfied. The explicit expressions for the components of $\mathcal{G}_{\mu}^{\nu}$ and $\mathcal{T}_{\mu}^{\nu}$ are given in the Appendix.

Consider first the equation $\mathcal{T}_{0}^{r}=0$. Assuming $h^{\prime} \neq 0$, this equation gives

$$
0=X \mathcal{F}_{X}+f_{1}\left(\mathcal{F}_{1}-2 f_{1} \mathcal{F}_{2}+3 f_{1}^{2} \mathcal{F}_{3}\right),
$$

where $\mathcal{F}_{X} \equiv \partial \mathcal{F} / \partial X$ and $\mathcal{F}_{i} \equiv \partial \mathcal{F} / \partial w_{i}$. Furthermore, the time and radial components of the energy-momentum tensor differ by the quantity proportional to eq. (5). Therefore, when this equation holds one has $\mathcal{T}_{0}^{0}=\mathcal{T}_{r}^{r}$. This implies that $\mathcal{G}_{0}^{0}=\mathcal{G}_{r}^{r}$ or, equivalently,

$$
\alpha(r) \beta(r)=1,
$$


in full analogy with the Schwarzschild solution in GR. Hence, the Einstein equations reduce to the following four equations,

$$
\begin{aligned}
1= & \alpha \beta, \\
0= & \frac{\alpha^{\prime}}{r}+\frac{\alpha-1}{r^{2}}-\frac{m^{2}}{2}\left(\mathcal{F}-2 X \mathcal{F}_{X}\right), \\
0= & \frac{\alpha^{\prime}}{r}+\frac{\alpha^{\prime \prime}}{2}-\frac{m^{2}}{2}\left[\mathcal{F}+X \mathcal{F}_{X}\right. \\
& \left.-w_{1} \mathcal{F}_{1}-2 w_{2} \mathcal{F}_{2}-3 w_{3} \mathcal{F}_{3}\right], \\
0= & X \mathcal{F}_{X}+f_{1}\left(\mathcal{F}_{1}-2 f_{1} \mathcal{F}_{2}+3 f_{1}^{2} \mathcal{F}_{3}\right),
\end{aligned}
$$

where $m^{2}=\Lambda^{4} / \mathrm{M}_{\mathrm{pl}}^{2}$. For a generic function $\mathcal{F}$, this system of equations is well defined. Indeed, since the function $h(r)$ enters the equations (6-9) only through the variable $X$, one may consider $X$ as an independent variable instead of $h(r)$. Then the fourth equation allows to find $\phi$ in terms of $X$, while the first equation gives $\beta$ in terms of $\alpha$. The second equation then gives $X$ in terms of $\alpha$ and the third equation allows to determine $\alpha$ as a function of $r$.

\section{Analytical example}

Finding analytical solutions of the non-linear system of equations like (6-9) is impossible for a generic function $\mathcal{F}$. So, in order to get some insight into the behavior of the solutions, let us choose the function $\mathcal{F}$ in such a way that the resulting equations are solvable analytically.

Consider the function $\mathcal{F}$ of the following form,

$$
\begin{aligned}
\mathcal{F}= & c_{0}\left(\frac{1}{X}+w_{1}\right) \\
& +c_{1}\left(w_{1}^{3}-3 w_{1} w_{2}-6 w_{1}+2 w_{3}-12\right),
\end{aligned}
$$

where $c_{0}$ is an arbitrary dimensionless constant and $c_{1}=$ \pm 1 (the numerical value of $c_{1}$ can be absorbed into the constant $\Lambda$ ). The coefficients inside the parentheses are chosen in such a way that the vacuum (2) is the solution to the Einstein equations at $a=b=1$. Our example contains, therefore, a single continuous free parameter $c_{0}$. Two additional constraints should be imposed on $c_{0}$. The first one comes from the requirement that the graviton is non-tachyonic. This translates into the inequality

$$
c_{0}-6 c_{1} \geq 0 .
$$

The second condition is necessary to ensure that scalar modes with pathological behavior do not reappear upon addition of higher-derivative terms (recall that the model (1) is understood as the low-energy effective theory, so such terms are generically present). This imposes certain constraint on the function $\mathcal{F}$ and its derivatives (cf. eq. (70) of Ref.[16]), which in the case at hand implies

$$
c_{0}>0 \text {. }
$$

We should stress that the choice of the functional form of $\mathcal{F}$ is by no means unique. The particular form (10) has been chosen in order to simplify the solution of the field equations, as will become clear below.

\subsection{The static spherically symmetric solution}

Let us start with eq. (9) which takes the form

$$
0=\frac{1}{X}\left[-c_{0}+\phi^{\prime 2}\left(6 c_{1} \frac{\phi^{4}}{r^{4}}+c_{0}-6 c_{1}\right)\right] .
$$

Because of our choice of the function $\mathcal{F}$, this equation contains the variable $X$ as an overall factor only. Hence, it reduces to a closed differential equation for $\phi$. The solution to this equation is

$$
\phi=b r
$$

where the constant $b$ satisfies the equation

$$
0=\left(b^{2}-1\right)\left(6 b^{4}+6 b^{2}+c_{0} / c_{1}\right) .
$$

We are interested in real positive values of $b$ (the case $b<0$ can be reduced to $b>0$ by the inversion of coordinates). If $c_{0} / c_{1}>0$ there is only one such solution,

$$
b=1 .
$$

If $c_{0} / c_{1}<0$ there exists another one,

$$
b=\frac{1}{\sqrt{2}}\left(-1+\sqrt{1-2 c_{0} / 3 c_{1}}\right)^{1 / 2} .
$$

Thus, at negative $c_{0} / c_{1}$ we have two different branches of solutions.

The remaining equations (7) and (8) can be written as follows,

$$
\begin{aligned}
& 0=\frac{\alpha^{\prime}}{r}+\frac{\alpha-1}{r^{2}}-3 \Lambda_{c}+m^{2} c_{0}\left(1-\frac{1}{X}\right), \\
& 0=\alpha^{\prime \prime}+\lambda \frac{\alpha-1}{r^{2}}+\left(\frac{\alpha^{\prime}}{r}-3 \Lambda_{c}\right)(2+\lambda),
\end{aligned}
$$

where

$$
\begin{aligned}
\lambda & =-12 b^{6} \frac{c_{1}}{c_{0}}, \\
\Lambda_{c} & =2 m^{2} c_{1}\left(b^{6}-1\right) .
\end{aligned}
$$

Recall that, according to our normalization, the constant $c_{1}$ only takes two values, $c_{1}= \pm 1$.

Eq. (14) is a linear inhomogeneous equation for $\alpha$. Its general solution can be found analytically. Making use of this solution and integrating the remaining equations one finally obtains:

$$
\begin{aligned}
\alpha(r) & =1-\frac{r_{s}}{r}-\frac{S}{r^{\lambda}}+\Lambda_{c} r^{2}, \\
\beta(r) & =1 / \alpha(r), \\
h(r) & = \pm \int \frac{\mathrm{d} r}{\alpha}\left[1-\alpha\left(\frac{S}{c_{0} m^{2}} \frac{\lambda-1}{r^{\lambda+2}}+1\right)^{-1}\right]^{1 / 2}, \\
\phi(r) & =b r .
\end{aligned}
$$

Here $r_{s}$ and $S$ are two integration constants: $r_{s}$ is the Schwarzschild radius, while $S$ is a scalar charge whose presence reflects the modification of the gravitational interaction as compared to GR. At $S=0$ the solution (15) 
reduces to the conventional Schwarzschild solution describing a black hole of the mass $M=r_{s} /\left(2 G_{N}\right)$.

The behavior of the metric at $r \rightarrow \infty$ depends on the constants $c_{0}$ and $c_{1}= \pm 1$. If $c_{1}=1$, we must also take $c_{0} \geq 6$, and the only solution of eq. (13) in this case is $b=1$. Hence, we have $\Lambda_{c}=0, \lambda<0$, and the metric is growing at infinity as $S r^{|\lambda|}$. Such solutions do not describe asymptotically flat space.

If $c_{1}=-1$, we must take $c_{0}>0$ in order to satisfy eqs. (11) and (12). In this case $\lambda>0$. Two branches of solutions exist, one with zero, and one with non-zero cosmological constant, which can be positive or negative depending on the numerical value of $c_{0}$. In what follows we consider the branch with $\Lambda_{c}=0(b=1)$.

The behavior of the solutions is determined by the two integration constants $M$ and $S$, and the value of the parameter $\lambda$. If $\lambda<1$, the third term on the r.h.s. of eq. (15) dominates at large distances. The ADM mass of these solutions is infinite. We do not discuss them further here.

If $\lambda>1$, the standard Schwarzschild term dominates at infinity. The ADM mass of such solutions is equal to $M$. The solutions with positive (negative) $M$ have attractive (repulsive) behavior at infinity.

At the origin $r=0$ both terms proportional to $M$ and $S$ are singular, so the metric always possesses a singularity unless $M=S=0$. This singularity may or may not be hidden by the horizon depending on the signs and values of $M$ and $S$. The solutions possessing the horizon are candidates for modified black holes.

The horizon is always present if both $M$ and $S$ are positive. Such black holes have attractive gravitational potential at all distances, which is stronger than for a conventional black hole of the mass $M$. The horizon size of the modified black hole is larger than $r_{s}=2 G_{N} M$.

If $M>0$ and $S<0$, the presence of the horizon depends on the relative values of $S$ and $M$. It exists for sufficiently small $S$. Defining, on dimensional grounds, the mass parameter $s$ associated with $S$ by the relation $|S|=s^{-\lambda}$, the existence of horizon requires that

$$
s M \geq \frac{\lambda}{2 G_{N}}\left(\frac{1}{\lambda-1}\right)^{\frac{\lambda-1}{\lambda}} .
$$

The Newtonian potentials for solutions satisfying and not satisfying the condition (16) are shown in Fig. 1-(a) and (b), respectively. When the horizon exists, the gravitational field is attractive all the way to the horizon. The attraction is weaker than in the case of the usual Schwarzschild black hole of mass $M$, and the horizon size is smaller. The behavior of the gravitational force with distance mimics that of the smaller-mass black hole plus a continuous distribution of "dark matter", with the total mass enclosed within the radius $r$ approaching $M$ as $r \rightarrow \infty$.

Finally, at $M<0$ and $S>0$ the modified black hole anti-gravitates at large distances and gravitates close to the horizon. The attraction changes to repulsion at

$$
r=r_{*} \equiv\left|\frac{\lambda S}{r_{s}}\right|^{\overline{\lambda-1}} .
$$

The corresponding Newtonian potential is shown in Fig. 1(c).

A remark is in order at this point. In the conventional general relativity the constant $r_{s}$ or equivalently, the black hole mass $M$, is also a free parameter which can, in principle, be positive or negative. In GR, however, only positive values make sense for the following reasons. First, negative-mass Schwarzschild solutions possess naked singularity at the origin, which is physically unacceptable. Second, the conventional matter satisfies the null energy condition which ensures that any compact spherically-symmetric matter distribution has a positive mass [31]. None of these arguments go through in the case of massive gravity. Fig. 1-(c) gives an example of solution with repulsive behavior at large distances and without naked singularity: as for a conventional black hole, the singularity of this solution is hidden behind the horizon. The positivity of energy is also not expected in massive gravity. This is related to the fact that the background (2) breaks time translations, and only the combination of the time translations with the shifts of $\phi^{0}$ by a constant remains unbroken. In this respect the massive gravity model is exactly analogous to the ghost condensate model [15], where the negative-energy states have been constructed explicitely [32].

\subsection{Correspondence with linear analysis}

The solutions found in the previous section have the asymptotic behavior different from that obtained in the linear perturbation theory in Ref. [20]. In order to compare our results with those of Ref. [20], let us discuss them in the gauge where $h(r)=0, \delta(r) \neq 0$ (cf. Sect. 3). In this gauge the perturbation theory of Ref. [20] corresponds to assuming that the variations of all the metric components are of the same order. In other words, they are formally assigned a small parameter $\epsilon$ to the first power. The solutions described in Ref. [20] satisfy the Einstein equations expanded to the linear order in $\epsilon$.

The solution (15) is not of this type. Transforming it into the gauge $h(r)=0$ one finds that $g_{t r}=\delta(r)$ does not decay as fast as the perturbations of other components, for instance, as $\alpha-1$. In fact, in the equations expanded in powers of perturbations the terms of order $\delta^{2}$ balance those linear in $\alpha-1$. In other words, in the formal expansion of the solution of Sect. 4 in powers of the small parameter $\epsilon$ the perturbation $\delta$ should be assigned the order $\sqrt{\epsilon}$ rather than $\epsilon$. Hence, the solution (15) is non-linear even at large distances from the center. A similar phenomenon has been observed in the context of bi-gravity models in Ref. [28].

Another difference between the solution (15) and the solution to the linearized equations of Ref. [20] is that the 


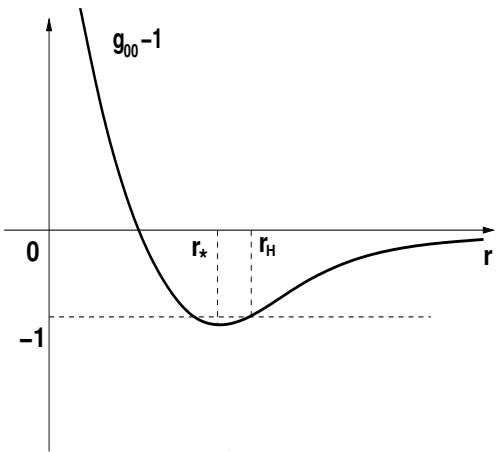

(a)

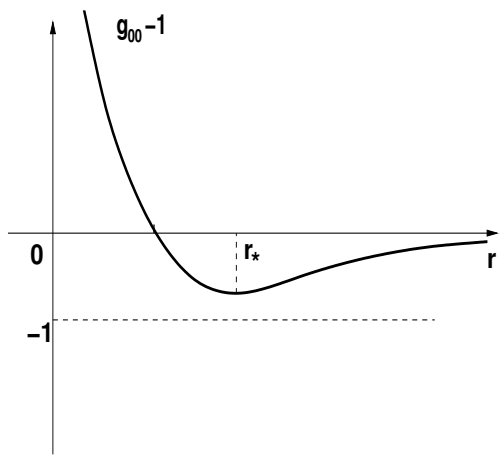

(b)

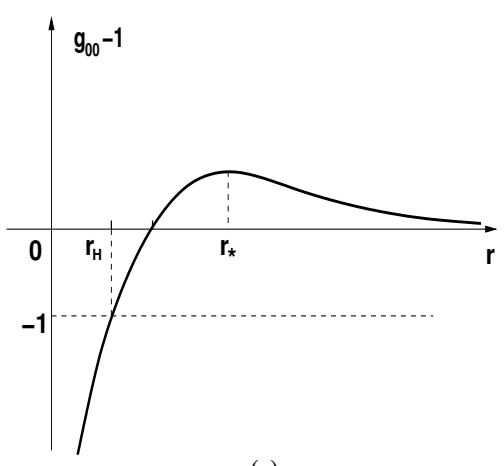

(c)

Figure 1: The deviation of $g_{00}$ from one (proportional to the Newtonian potential $\left.\Phi=\left(g_{00}-1\right) / 2\right)$ for tree different choices of the integration constants. Figs. (a) and (b) correspond to $M>0$ and $S<0$ with the numerical values satisfying (a) and not satisfying (b) eq. (16). Fig. (c) represents solution with $M<0$ and $S>0$.

former is static, while in the latter only metric components are static (in the gauge $g_{0 i}=0$ ). The scalar fields have time dependence which may be viewed as an accretion of a fluid with zero energy-momentum tensor.

\subsection{Gravitational field of a star}

In general relativity, one may relate the mass of a star to an integration constant of the vacuum solution in the exterior space by matching the interior and exterior solutions at the star surface (see, e.g., Ref. [33]). In massive gravity, one may try to use the same approach to determine the scalar charge of an ordinary star. The analytical solution in the interior region is required for the matching procedure.

The star is described, to a good approximation, by a diagonal energy-momentum tensor $t_{\mu}^{\nu}=(\rho,-p,-p,-p)$, where $\rho$ and $p$ are the energy density and pressure inside the star, respectively. This energy-momentum tensor is assumed to be responsible for the external gravitational field described by eq. (15). Since there is no direct coupling between the ordinary matter and the Goldstone fields, $t_{\mu}^{\nu}$ must be conserved separately, $\nabla^{\mu} t_{\mu}^{\nu}=0$. For simplicity, we take the energy density to be constant at $r<R$, where $r=R$ is the surface of the star, and zero outside. The pressure $p$ cannot be chosen independently; it is determined by the conservation of $t_{\mu}^{\nu}$.

Because of the spherical symmetry, the ansatz (4) holds. The Einstein equations in the interior of the star are obtained from eqs. (6-8) by adding the contributions of the energy-momentum of the star, while eq. (9) remains unchanged. The resulting set of equations can be solved an- alytically. The solution reads

$$
\begin{aligned}
\alpha(r)= & 1-\frac{s_{1}}{r}-\frac{s_{2}}{r^{\lambda}}+\Lambda_{c} r^{2} \\
& +\frac{\rho}{\mathrm{M}_{\mathrm{pl}}^{2}}\left(\frac{r^{2}}{6}-\frac{R^{2}}{2}\right)+\mathcal{O}\left(\rho^{2}\right), \\
\beta(r)= & {\left[1-\frac{s_{1}}{r}-\frac{s_{2}}{r^{\lambda}}+\Lambda_{c} r^{2}-\frac{r^{2} \rho}{3 \mathrm{M}_{\mathrm{pl}}^{2}}\right]^{-1}, } \\
h(r)= & \pm \int \frac{\mathrm{d} r}{\alpha}\left[1-\alpha\left(\frac{s_{2}}{c_{0} m^{2}} \frac{\lambda-1}{r^{\lambda+2}}+1\right)^{-1}\right]^{1 / 2}, \\
\phi(r)= & b r .
\end{aligned}
$$

For simplicity, we have expanded the first equation in powers of $\rho$, while the other relations are exact. Since the geometry inside the star is regular, the integration constants $s_{1}$ and $s_{2}$ must be set to zero.

The interior solution has to be matched with the solution (15) at $r=R$. It is convenient to match the variable $X$ which equals 1 in the interior region. In the gauge $h(r)=0$ this variable is nothing but the $g^{00}$ component of the metric. Hence, it must be continuous. Making use of eqs. (15) one can see that the continuity of $X$ at $r=R$ requires that $S=0$. Therefore, the scalar charge of an ordinary star is zero.

It remains an open question how objects (e.g., black holes) with $S \neq 0$ can be created. The argument given above does not apply to time-dependent configurations, so it is possible that a non-zero scalar charge may be acquired during the gravitational collapse.

\section{5. $\mathcal{F}\left(Z^{i j}\right)$ models}

As mentioned earlier, models characterized by the function $\mathcal{F}$ of a single variable $Z^{i j}=X^{\gamma} W^{i j}$ are of a particular interest. We discuss in this section the exact static spherically symmetric solutions in these models. Our goal is to 
demonstrate that the solutions found earlier are not specific to the particular form of the action (10) and exist also in the models obeying the symmetry (3).

In Sect. 4 the analytical solutions of eqs. (6-9) were obtained by choosing the function $\mathcal{F}$ in such a way that the dependence on $X$ factors out in eq. (9). Since now $\mathcal{F}$ has only one argument, the derivatives of $\mathcal{F}$ with respect to $X$ and $W^{i j}$ are no more independent. For this reason we did not succeed in constructing non-trivial examples where the Einstein equations are solvable analytically. Hence, to demonstrate the existence of solutions we have to use numerical methods.

Consider the following function $\mathcal{F}$,

$$
\begin{aligned}
\mathcal{F}= & c_{0}\left(z_{1}+2+\frac{z_{1}^{3}-6 z_{1} z_{2}+8 z_{3}}{3}\right) \\
& +2 c_{1}\left(z_{1}^{2}-2 z_{2}-1-2 \frac{z_{1}^{3}-6 z_{1} z_{2}+8 z_{3}}{3}\right),
\end{aligned}
$$

were $z_{n}=\operatorname{Tr}\left(Z^{n}\right)$ are tree independent scalars made out of $Z^{i j}, z_{n}=\left(X^{\gamma}\right)^{n} w_{n}$. The coefficients in front of individual terms have been adjusted so that the flat metric and the scalar fields given by eq. (2) solve the field equations at $a=b=1$. For the vacuum configuration (2) one has $Z^{i j}=-\delta^{i j}$. We are interested in solutions to the field equations that asymptote to this vacuum state.

In addition to the adjustments already made, the following inequality should be imposed on the coefficients $c_{0}$ and $c_{1}$ to ensure that the graviton is non-tachyonic,

$$
c_{0}-2 c_{1} \geq 0 .
$$

This guarantees that the square of the graviton mass is non-negative. Moreover, this inequality is sufficient for the absence of pathological scalar modes which may appear upon addition of higher-derivative terms. As in the previous example, the overall scale of the coefficients $c_{0}$ and $c_{1}$ can be absorbed in the parameter $\Lambda$, so without loss of generality we may set $c_{1}= \pm 1$.

For this class of models, the field equations (6-9) may be viewed as equations for $\alpha(r), \beta(r), \xi(r) \equiv X^{\gamma} f_{1}-1$ and $\psi(r) \equiv X^{\gamma} f_{2}-1$. Then eq. (6) gives $\beta$ in terms of $\alpha$, while eq. (9) enables to express $\psi$ in terms of $\xi$. The two remaining equations form a coupled set of non-linear equations for $\alpha$ and $\xi$; they have to be solved numerically.

The numerical solutions are shown in Fig. 2 for different value of the parameters $c_{0}$ and $c_{1}$. For all these graphs, we have assumed that the external horizon is located at $r=1$ and that $\xi=100$ at the horizon. The large value of $\xi$ is chosen in order to make the difference between the modified solution and the Schwarzschild solution visible on the plot (large values of $\xi$ correspond to large scalar charge $S$ of the previous section).

The plots show the behavior qualitatively similar to that discussed in Section 4. In particular:

- if $c_{0}=c_{1}=-1$, the Newtonian potential $2 \Phi=\alpha-1$ is attractive at short distances and becomes repulsive at larger distances;

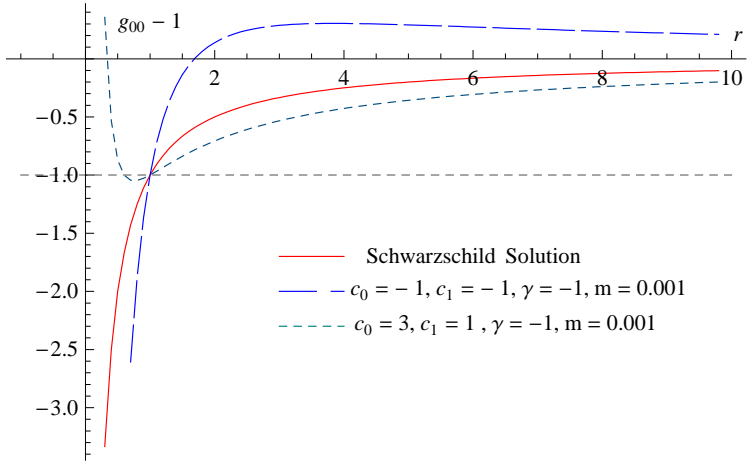

Figure 2: The deviation of $g_{00}$ from one for three different cases: the usual Schwarzschild solution (solid line) and two solutions corresponding to different values of the parameters of the function (17) (long-dashed and short-dashed lines). The integration constants of these solutions have been chosen such that the external horizon is located at $r=1$.

- if $c_{0}=3$ and $c_{1}=1$, the Newtonian potential is attractive outside of the horizon, and becomes repulsive close to the singularity.

The deviations from the Schwarzschild metric are larger for larger values of the "scalar charge" (parameterized by the value of $\xi$ at the horizon). The Schwarzschild solution is recovered at $\xi \rightarrow 0$.

\section{Discussion}

To summarize, there exist spherically symmetric vacuum solutions in massive gravity models which depend on two integration constants, the mass $M$ and an extra parameter $S$ which can be called the "scalar charge". At zero value of the scalar charge the standard Schwarzschild solution is recovered, while at non-zero $S$ the metric is modified with respect to the Schwarzschild case.

The solutions having non-zero scalar charge exhibit much reacher behavior than the Schwarzschild solution in GR. As can be seen from the explicit example of Sect. 4, both the short and long distance behavior may be modified depending on the parameters of the model.

Unlike in General Relativity, the solutions may have a negative ADM mass. Such solutions have repulsive gravitational interaction at large distances. At short distances the repulsion may change to attraction and give rise to the horizon, hiding the singularity at the origin. Such solutions represent anti-gravitating black holes.

In the case of a positive ADM mass, the $S$-dependent contributions may make the gravitational attraction weaker at short distances (cf. fig. 1-(a)). In this case the gravitational force decays with distance slower than $1 / r^{2}$, thus mimicking the presence of dark matter. Interestingly, solutions with the same value of $M$ but different scalar charge $S$ have different behavior, which corresponds to different amount of the apparent "dark matter". This 
is in contrast with other models possessing modifications of the gravitational potential $[4,6,10]$, where the modification of the gravitational force is determined by the parameters of the model.

It is currently an open question how objects with nonzero scalar charge may be created. As has been argued in Sect. 4.3, the absence of direct coupling between the Goldstone fields and ordinary matter results in zero scalar charges of static matter distributions. Thus, the gravitational field of ordinary stars is described by the $S=0$ solutions, i.e., by the standard Schwarzschild metric. This may be not the case for black holes, especially the supermassive black holes in the centers of galaxies, which may be of primordial origin $[34,35]$. In any case, this question requires further investigation.

Another open question is the stability of the modified black hole solutions. Several kinds of instabilities may be present. Among perturbations of the solutions there may exist unstable modes with the characteristic time scale of order of the horizon size; in this case the interpretation in terms of black holes is not possible. Second potential source of problems is generic presence of the higherderivative terms not included in the action (1). One has to check that the 2-parameter family of modified black holes survives their inclusion. By analogy with the ghost condensate case, one may expect that these terms produce at least a slow Jeans-type instability [15], which is not, however, dangerous for the black hole interpretation. Finally, the presence of negative mass solutions may lead to instabilities of the quantum-mechanical nature similar to those found in Ref.[36].

To conclude this list, let us mention also the solutions satisfying $h=0$ which were not considered in this paper. In this case the ten Einstein equations reduce to three equations for $\alpha, \beta$ and $\phi$ which form a (generically) welldefined system. It remains to be seen whether this system has asymptotically flat solutions. In any case, the Schwarzschild solution does not belong to this class which is characterized by $\alpha \beta \neq 1$.

\section{Acknowledgments}

We are grateful to S. Dubovsky for valuable discussions and comments on the manuscript. The work of M.B. is supported by the Belgian Fond pour la Formation à la Recherche dans l'Industrie et dans l'Agriculture (FRIA). The work of P.T. is supported by IISN, Belgian Science Policy (under contract IAP V/27).

\section{Appendix}

For the ansatz (4) discussed in Section 3, the non-zero components of the Einstein tensor are given by the follow- ing relations

$$
\begin{aligned}
\mathcal{G}_{0}^{0} & =\frac{1}{r^{2}}\left[1-\left(\frac{r}{\beta}\right)^{\prime}\right], \\
\mathcal{G}_{r}^{r} & =\frac{1}{r^{2}}\left(1-\frac{\alpha+r \alpha^{\prime}}{\alpha \beta}\right), \\
\mathcal{G}_{\theta}^{\theta} & =\mathcal{G}_{\varphi}^{\varphi}=-\frac{1}{4 r}\left[\frac{\alpha^{\prime}+r \alpha^{\prime \prime}}{\alpha \beta}+\left(\frac{2 \alpha+r \alpha^{\prime}}{\alpha \beta}\right)^{\prime}\right] .
\end{aligned}
$$

The energy-momentum tensor of the Goldstone fields is expressed through

$$
\begin{aligned}
\mathcal{T}_{\mu}^{\nu}= & -\frac{1}{2} \Lambda^{4} \delta_{\mu}^{\nu} \mathcal{F}+\mathcal{F}_{X} \partial^{\nu} \phi^{0} \partial_{\mu} \phi^{0} \\
& +\frac{\partial \mathcal{F}}{\partial W^{i j}}\left[\partial^{\nu} \phi^{i} \partial_{\mu} \phi^{j}+\frac{V^{i} V^{j}}{X^{2}} \partial^{\nu} \phi^{0} \partial_{\mu} \phi^{0}\right. \\
& \left.-\frac{V^{j}}{X}\left(\partial^{\nu} \phi^{i} \partial_{\mu} \phi^{0}+\partial^{\nu} \phi^{0} \partial_{\mu} \phi^{i}\right)\right]
\end{aligned}
$$

where $\mathcal{F}_{X} \equiv \partial \mathcal{F} / \partial X$ and

$$
V^{i}=\frac{\partial^{\mu} \phi^{0} \partial_{\mu} \phi^{i}}{\Lambda^{4}}
$$

For functions $\mathcal{F}$ which are invariant under rotations of the Goldstone fields $\phi^{i}$ internal space, the derivatives of $\mathcal{F}$ with respect to $W^{i j}$ are given by

$$
\frac{\partial \mathcal{F}}{\partial W^{i j}}=\mathcal{F}_{1} \delta_{i j}+2 \mathcal{F}_{2} W^{i j}+3 \mathcal{F}_{3} W^{i k} W^{k j},
$$

where $\mathcal{F}_{i} \equiv \partial \mathcal{F} / \partial w_{i}$. Therefore, the components of the energy-momentum tensor which are not identically zero are given by

$$
\begin{aligned}
\mathcal{T}_{0}^{0}= & \Lambda^{4}\left[-\frac{1}{2} \mathcal{F}+\frac{1}{\alpha}\left(\mathcal{F}_{X}+\frac{\partial \mathcal{F}}{\partial W^{i j}} \frac{V^{i} V^{j}}{X^{2}}\right)\right], \\
\mathcal{T}_{r}^{r}= & \Lambda^{4}\left[-\frac{1}{2} \mathcal{F}-\left(\mathcal{F}_{X}+\frac{\partial \mathcal{F}}{\partial W^{i j}} \frac{V^{i} V^{j}}{X^{2}}\right) \frac{h^{\prime 2}}{\beta}\right] \\
& +\frac{1}{\beta} \frac{\partial \mathcal{F}}{\partial W^{i j}}\left(-\partial_{r} \phi^{i} \partial_{r} \phi^{j}+\frac{2 V^{j}}{X} \partial_{r} \phi^{i} \partial_{r} \phi^{0}\right), \\
\mathcal{T}_{\theta}^{\theta}= & \mathcal{T}_{\varphi}^{\varphi}=-\frac{1}{2} \Lambda^{4} \mathcal{F}+\frac{\partial \mathcal{F}}{\partial W^{i j}} \partial^{\theta} \phi^{i} \partial_{\theta} \phi^{j} \\
\mathcal{T}_{0}^{r}= & -\frac{\Lambda^{4} h^{\prime}}{\beta}\left[\mathcal{F}_{X}+\frac{\partial \mathcal{F}}{\partial W^{i j}}\left(\frac{V^{i} V^{j}}{X^{2}}+\frac{\partial_{r} \phi^{i} \partial_{r} \phi^{j}}{\beta \Lambda^{4} X}\right)\right] .
\end{aligned}
$$

\section{References}

[1] Supernova Cosmology Project, S. Perlmutter et al., Astrophys. J. 517, 565 (1999), astro-ph/9812133.

[2] WMAP, J. Dunkley et al., (2008), 0803.0586.

[3] SDSS, J. K. Adelman-McCarthy, Astrophys. J. Suppl. 172, 634 (2007), 0707.3380.

[4] M. Milgrom, Astrophys. J. 270, 371 (1983).

[5] J. D. Bekenstein, Phys. Rev. D70, 083509 (2004), astroph/0403694.

[6] G. R. Dvali, G. Gabadadze, and M. Porrati, Phys. Lett. B485, 208 (2000), hep-th/0005016.

[7] R. Gregory, V. A. Rubakov, and S. M. Sibiryakov, Phys. Rev. Lett. 84, 5928 (2000), hep-th/0002072. 
[8] T. Damour and I. I. Kogan, Phys. Rev. D66, 104024 (2002), hep-th/0206042.

[9] D. Blas, C. Deffayet, and J. Garriga, Phys. Rev. D76, 104036 (2007), 0705.1982.

[10] S. M. Carroll, V. Duvvuri, M. Trodden, and M. S. Turner, Phys. Rev. D70, 043528 (2004), astro-ph/0306438.

[11] G. Dvali, O. Pujolas, and M. Redi, Phys. Rev. Lett. 101, 171303 (2008), 0806.3762.

[12] G. Gabadadze, (2003), hep-ph/0308112.

[13] V. A. Rubakov and P. G. Tinyakov, Phys. Usp. 51, 759 (2008), 0802.4379.

[14] T. P. Sotiriou and V. Faraoni, (2008), 0805.1726.

[15] N. Arkani-Hamed, H.-C. Cheng, M. A. Luty, and S. Mukohyama, JHEP 05, 074 (2004), hep-th/0312099.

[16] S. L. Dubovsky, JHEP 10, 076 (2004), hep-th/0409124.

[17] V. A. Rubakov, (2004), hep-th/0407104.

[18] S. L. Dubovsky, P. G. Tinyakov, and I. I. Tkachev, Phys. Rev. D72, 084011 (2005), hep-th/0504067.

[19] J. H. Taylor and J. M. Weisberg, Astrophys. J. 253, 908 (1982).

[20] S. L. Dubovsky, P. G. Tinyakov, and I. I. Tkachev, Phys. Rev. Lett. 94, 181102 (2005), hep-th/0411158.

[21] M. V. Bebronne and P. G. Tinyakov, Phys. Rev. D76, 084011 (2007), 0705.1301.

[22] McClintock, J. E. and Remillard, R. A., Astrophys. J. 308, 110 (1986).

[23] J. E. McClintock and R. A. Remillard, (2003), astroph/0306213.

[24] R. Genzel, A. Eckart, T. Ott, and F. Eisenhauer, Mon. Not. Roy. Astron. Soc. 291, 219 (1997).

[25] S. Gillessen et al., (2008), 0810.4674.

[26] C. M. Will, Living Rev. Rel. 9, 3 (2005), gr-qc/0510072.

[27] S. Dubovsky, P. Tinyakov, and M. Zaldarriaga, JHEP 11, 083 (2007), 0706.0288.

[28] Z. Berezhiani, D. Comelli, F. Nesti, and L. Pilo, (2008), 0803.1687.

[29] R. L. Arnowitt, S. Deser, and C. W. Misner, Phys. Rev. 122, 997 (1961).

[30] M. V. Bebronne, Phys. Lett. B668, 432 (2008), 0806.1167.

[31] S. W. Hawking and G. F. R. Ellis, (1973), Cambridge University Press, Cambridge.

[32] N. Arkani-Hamed, H.-C. Cheng, M. A. Luty, S. Mukohyama, and T. Wiseman, JHEP 01, 036 (2007), hep-ph/0507120.

[33] S. Weinberg, Gravitation and Cosmology : Principles and Applications of the General Theory of Relativity (Wiley, 1972).

[34] B. J. Carr and S. W. Hawking, Mon. Not. Roy. Astron. Soc. 168, 399 (1974).

[35] B. J. Carr, (2005), astro-ph/0504034.

[36] D. Krotov, C. Rebbi, V. A. Rubakov, and V. Zakharov, Phys. Rev. D71, 045014 (2005), hep-ph/0407081. 